\title{
NUMERICAL METHODS FOR ANISOTROPIC MEAN CURVATURE FLOW BASED ON A DISCRETE TIME VARIATIONAL FORMULATION*
}

\author{
ADAM OBERMAN ${ }^{\dagger}$, STANLEY OSHER $^{\ddagger}$, RYO TAKEI $^{\S}$, AND RICHARD TSAI $₫$
}

\begin{abstract}
Numerical methods for planar anisotropic mean curvature flow are presented for smooth and crystalline anisotropies. The methods exploit the variational level-set formulation of A. Chambolle, in conjunction with the split Bregman algorithm (equivalent to the augmented Lagrangian method and the alternating directions method of multipliers). This induces a decoupling of the anisotropy, resulting in a linear elliptic PDE and a generalized shrinkage (soft thresholding) problem. In the crystalline anisotropy case, an explicit formula for the shrinkage problem is derived. In the smooth anisotropy case, a system of nonlinear evolution equations, called inverse scale space flow, is solved. Numerical results are presented.
\end{abstract}

Key words. Anisotropic mean curvature flow, Wulff shapes, total variation minimization, split Bregman method, shrinkage, soft thresholding, inverse scale space.

AMS subject classifications. 35K65, 35K55, 65M06, 65M12, 65K10, 49M25.

\section{Introduction}

We study numerical methods for the time evolution of a closed planar curve $C(t)$ with normal velocity equal to its anisotropic mean curvature. The motion is the gradient flow of the anisotropic perimeter

$$
\mathcal{E}_{\phi}(C)=\int_{C} \phi(\hat{n}) d s
$$

Here, $\hat{n}$ is the unit outer normal to the curve $C$ and $d s$ is the arc-length measure. For a derivation, we refer to the survey [48] and the references therein. In the case $\phi(\cdot)=|\cdot|$, the motion is the well known (isotropic) mean curvature flow, or the curve-shortening flow, where the curve evolves to minimize its length.

The direct approach is to solve the gradient flow of the surface free energy for a given parameterized curve. The formulation is as follows: let $\eta(C)$ be a small deformation of a closed curve $C$. Then, for a 'time step' $h>0$, find the particular deformation $\eta^{*}$ that minimizes

$$
\min _{\eta} \mathcal{E}_{\phi}(\eta(C))-\mathcal{E}_{\phi}(C)+\frac{1}{2 h}\langle\eta, \eta\rangle
$$

It can be shown (c.f. [48]) that, $\eta^{*}(C)$ is an approximation to the anisotropic mean curvature evolution of $C$ by time $h$. This variational approach was studied in $[1,35$, $13]$.

The level set method (see Section 1.2) can also be used to implement isotropic and smoothly anisotropic motions. It cannot be used in the crystalline case because the motion is nonlocal. However, it is possible to combine the advantages of the level set approach with the variational formulation. Chambolle [9] showed that minimizing

*Received: May 16, 2010; accepted (in revised version): November 10, 2010. Communicated by Peter Smereka.

${ }^{\dagger}$ Department of Mathematics, Simon Fraser University, Canada (aoberman@sfu.ca).

${ }^{\ddagger}$ Department of Mathematics, University of California, Los Angeles, USA (sjo@math.ucla.edu).

$\S$ Department of Mathematics, University of California, Los Angeles, USA (rrtakei@ucla.edu).

ๆ Department of Mathematics, University of Texas, Austin, USA (ytsai@math.utexas.edu). 
(1.2) can be achieved by defining $f$ to be the signed distance function to the curve $C$, and performing the minimization

$$
\min _{u \in L^{2}(\Omega)} \int_{\Omega} \phi(\nabla u)+\frac{1}{2 h}\|u-f\|_{L^{2}(\Omega)}^{2} .
$$

The first term is the anisotropic total variation; see the definition (2.3) below. If $u^{*}$ is the minimizer of (1.3), then $\eta^{*}(C)=\left\{u^{*}(x)=0\right\}$, the zero level set of $u^{*}$.

1.1. Contribution of this work. In this article, we consider two different scenarios of (1.3): one involving a non-smooth $\phi$ and the other with smooth but anisotropic $\phi$. We propose and implement two numerical algorithms for these two scenarios. The first numerical method treats crystalline anisotropies, and takes advantage of an explicit formula we derive in this case. Crystalline anisotropies are of interest, for example, in crystal growth; c.f. [32]. The second numerical method treats smooth anisotropies and yields a theoretically interesting result relating the level set and variational approaches.

To alleviate the difficulty in solving the nonlinear (and singular in the case of crystalline curvatures) minimization problem that arises from (1.3), we propose to deal with it by extensions of the split Bregman method, which solves (1.3) via a sequence of easier-to-solve problems. The crystalline anisotropy case is treated by modifying the split Bregman based algorithm [31] applied to isotropic mean curvature flow: in particular, we characterize the solution of the anisotropic shrinkage (or soft thresholding) problem: given $y \in \mathbb{R}^{2}$, find

$$
\arg \min _{x \in \mathbb{R}^{2}} \phi(x)+\frac{1}{2}|x-y|^{2} .
$$

In Theorem 4.3, we derive a formula for the minimizer of (1.4); embedding this formula within the split Bregman algorithm gives rise to the corresponding anisotropic mean curvature flow. The main advantage of this approach is that it circumvents the difficulty of treating the non-smoothness of $\phi$ by confining the anisotropy only within the subproblem (1.4); this observation was mentioned briefly in [25], but an explicit characterization is novel. While our solution to (1.4) is simple, an analytically explicit formula for general (e.g. smooth) $\phi$ is unavailable. Due to the convexity of the problem, one may employ efficient numerical methods to solve (1.4); we will comment on this later in Section 5.

For the smooth anisotropy case, we propose to solve a system of nonlinear equations, called an inverse scale space flow. This is inspired by the work of Burger et al. [3], where they interpret the iterative refinement of the Rudin-Osher-Fatemi (ROF) image denoising scheme as a discretization of a continuous evolution equation. In their context, the evolution initially captures large scale artifacts of a given noisy image $f$, then gradually finer scales are captured, eventually converging to $f$. For the present mean curvature problem, we apply the same strategy but to the split Bregman functional (5.4); to our knowledge, this application is new. The resulting inverse scale space flow share several properties to that of Burger et al. (e.g. decay of Bregman distance, result 6 of Lemma 5.3), but is distinct in that it evolves two functions that converge to each other in the limit. It is also of theoretical interest that the (nonlinear) evolution equations involves the linear term $\Delta u$, rather than the degenerate elliptic curvature $\operatorname{div}(\nabla u /|\nabla u|)$, a favorable property inherited from the split Bregman framework. We also show that the limit of the evolution is in fact the 
updated solution to a semi-implicit Euler scheme for the mean curvature partial differential equation (PDE); see Remark 5.1. Convergence proofs and numerical results are given.

For a brief outline of the proposed method in the context of the split Bregman method, see Section 2.2.

1.2. Motion of level sets by mean curvature. An alternative method for the direct minimization of the anisotropic perimeter (1.1) is to use the level set approach of Osher and Sethian [42]. The advantages of the level set approach are well documented: it naturally handles topological changes and can be implemented on a uniform, Cartesian grid. Note, however, for non-smooth surface free energies, such as the crystalline case, the level set method cannot be used since the motion is nonlocal [1]. (See (1.8), the term $\gamma(\omega)+\gamma^{\prime \prime}(\omega)$ is undefined in the crystalline case).

Represent the curve $C(t) \subset \mathbb{R}^{2}$ as the zero level set of an auxiliary function $u(x, t)$, $x \in \mathbb{R}^{2}$. Then, the idea is to evolve $u$ by a degenerate elliptic partial differential equation (PDE)

$$
\frac{\partial u}{\partial t}=|\nabla u| \operatorname{div} \frac{\nabla u}{|\nabla u|} .
$$

In fact, all level curves $\left\{x \in \mathbb{R}^{2}: u(x, t)=\right.$ constant $\}$ evolve by mean curvature under (1.5). This PDE was analyzed in the viscosity solutions framework in [22, 8].

The anisotropic analogue of (1.5) may take various forms. The general form is,

$$
\frac{\partial u}{\partial t}=\beta\left(\frac{\nabla u}{|\nabla u|}\right)|\nabla u| \operatorname{div} \nabla \phi(\nabla u)
$$

where $\beta$ is called the mobility function. It has been shown $[28,16]$ that this PDE produces self-similar solutions in two dimensions for positive, continuous $\beta$. Two special cases have been studied previously: the variational approach of Almgren, Taylor and Wang [1] solved the case $\beta=1$, while Chambolle [9] and Novaga and Paulini [38] studied the case $\beta=\gamma$, where $\gamma(x)=\phi(x) /|x|$. We chose to describe our methods for the anisotropic form

$$
\frac{\partial u}{\partial t}=|\nabla u| \operatorname{div} \nabla \phi(\nabla u),
$$

i.e. the case $\beta=1$, mainly to simplify the implementation, but other choices for the mobility function can be implemented by the present algorithms similarly; see remarks 4.2 and 5.2 .

Let us write,

$$
\phi(\nabla u)=\gamma(\omega)|\nabla u|, \quad \omega=\tan ^{-1}\left(u_{y} / u_{x}\right) .
$$

If $\gamma$ is smooth, (1.7) can be written as

$$
\frac{\partial u}{\partial t}=\left(\gamma(\omega)+\gamma^{\prime \prime}(\omega)\right)|\nabla u| \operatorname{div} \frac{\nabla u}{|\nabla u|} .
$$

The condition $\gamma(\omega)+\gamma^{\prime \prime}(\omega)>0$ to avoid ill-posedness is a standard assumption in interface motion; c.f. [32, Chapter 9]. The PDE (1.8) for smooth $\phi$ is still solvable numerically, for example, using conventional, explicit finite difference schemes. We will see in Section 5.3 (Proposition 5.2) that the inverse scale space flow approximates a discretization of (1.8) in $t$. 
1.3. Related work. In this section we discuss related work.

Theoretical work on anisotropic mean curvature flow have been studied extensively; we refer the interested reader to the survey article of Bellittini [2] (on crystalline mean curvature flows), the book by Giga [27] (on various interface problems, in the context of the level set equations) and the references therein.

Several numerical methods for Chambolle's approach have been studied. In the case $\phi=|\cdot|,(1.3)$ is the Rudin-Osher-Fatemi (ROF) functional used for image denoising [44]. Numerical methods based in the variational approach to mean curvature flow have recently gained attention due to the advent of fast ROF (minimization) solvers combined with efficient methods for computing distance functions. Chambolle and Darbon [6] used a combinatorical ROF solver, referred to as 'graph-cuts', to solve anisotropic mean curvature flow; the algorithm is designed only for crystalline anisotropies with the singularities aligned along grid directions. Chambolle [9] also proposed a ROF solver [10], a fixed point method of a projection in the dual space, for isotropic and crystalline anisotropies (in the latter case, numerical results were presented without proof of convergence). For the crystalline case, the present paper follows [25] where the split Bregman method [31] is used to solve the ROF minimization.

Numerical methods for the direct solution of the level set PDE for mean curvature flow have also been studied. Monotone, convergent finite difference schemes for the isotropic mean curvature motion PDE (1.5) were discovered by Catte, et al. [7] and Oberman [40]. Clarenz, et al. [11] considered a weak formulation of a ( $\epsilon$-regularized) level set equation for anisotropic mean curvature motion and solved it using a finite element scheme; later this technique was generalized to higher order flows in [5]. In the survey article [15], Deckelnick, et al. considered a semi-implicit Euler scheme of the finite element formulation of (1.5) divided by $|\nabla u|$ :

$$
\frac{1}{\left|\nabla u^{k}\right|} \frac{u^{k+1}-u^{k}}{h}=\operatorname{div} \frac{\nabla u^{k+1}}{\left|\nabla u^{k}\right|}
$$

where $h>0$ is the time step and $u^{k}(x)$ is an approximation to $u(x, t=k h)$. Note that in (1.9), the curvature term is approximated using both $u^{k}$ and $u^{k+1}$, while our proposed scheme, the curvature term is approximated only using the values of $u^{k}$; see Remark 5.1.

The Merriman-Bence-Osher (MBO) scheme [37] is a theoretical method for approximating isotropic motion by mean curvature. It approximates a mean curvature flow by repeatedly solving the heat equation for a short time and thresholding. Generalization to anisotropic flow has been studied in $[12,34]$. In a series of papers, $[23,19,20,21]$, Esedoglu et al. proposed a new class of algorithms for the computation of geometric motions of interfaces, including isotropic curvature motions and higher order motions such as motion by surface diffusion. These algorithms can be seen as generalizations of Merriman, Bence, and Osher's threshold dynamics, and consist of two alternating steps: the construction of either a characteristic function or a signed distance function to the interface, and convolution with a suitable kernel. In particular, by using signed distance functions, the algorithms proposed in [21] allow for high accuracy on uniform grids. Furthermore, motion by smooth anisotropic curvatures can also be computed after a slight modification of the first step. These algorithms preserve the computational efficiency of the Merriman-Bence-Osher scheme; in comparison to the conventional level set methods, they avoid the evaluation of degenerate nonlinear PDEs; in comparison to Chambolle's algorithm, they avoid the 
computation of nontrivial optimization problems. In this regard, the present article's contribution lies in alleviating the complexity for solving the nontrivial optimization problems in the framework of Chambolle. For other generalizations of the MBO scheme; see [45, 43, 26].

Hausser and Voigt [33] considered a regularized mean curvature flow by adding $\epsilon \kappa^{2}$ ( $\kappa$ is curvature and $\epsilon \ll 1$ ) to the interfacial energy (1.1); the evolution of the interface in two dimensions was solved via a marker-point type scheme; c.f. [46, II.3.1].

\section{Definitions and outline of main algorithms}

2.1. Definitions and notations. Throughout this article, let $\Omega$ be a bounded, connected, open subset of $\mathbb{R}^{2}$.

We start by defining $\phi$, the surface free energy function (using a term from thermomechanics [32]). Assume

$$
\begin{aligned}
& \phi \text { is a convex, even, positively } 1 \text {-homogeneous function } \\
& \text { with } 0<a(x) \leq \phi(x) \leq A(x)<\infty \text {, for all } x \in \mathbb{R}^{2} .
\end{aligned}
$$

The unit ball of the surface free energy function is known as the Frank diagram:

$$
\mathcal{F}_{\phi}:=\left\{y \in \mathbb{R}^{2} \mid \phi(y) \leq 1\right\}
$$

Also define $\phi^{\circ}$, the dual norm (or polar) of $\phi$ :

$$
\phi^{\circ}(x):=\sup _{\phi(y) \leq 1} x \cdot y .
$$

The Wulff shape of $\phi$ is the unit ball of $\phi^{\circ}$ :

$$
\mathcal{W}_{\phi}:=\left\{y \in \mathbb{R}^{2}: \phi^{\circ}(y) \leq 1\right\} .
$$

Note that, by the convexity of $\phi$, the Wulff shape $\mathcal{W}_{\phi}$ is convex. In addition, the Wulff shape and Frank diagram are related by duality:

$$
\phi(x)=\sup _{\phi^{\circ}(y) \leq 1} y \cdot x=\sup _{y \in \mathcal{W}_{\phi}} y \cdot x .
$$

EXAMPLE 2.1. A standard example of a dual pair of norms is the 1-norm $|\cdot|_{1}$ and the max-norm $|\cdot|_{\infty}$. For $p>1$, the dual norm of the $p$-norm $|\cdot|_{p}$ is the $q$-norm with $1 / p+1 / q=1$. For a positive definite invertible matrix $A$, the norm $\phi(x)=|A x|$ has the dual $\phi(x)=\left|A^{-T} x\right|$. See Appendix A for general formulas of Wulff shapes for a special class of $\phi$, i.e. polyhedral norms.

Define the (isotropic) total variation of a function $u \in L_{l o c}^{1}(\Omega)$ to be

$$
\int_{\Omega}|\nabla u|:=\sup \left\{\int_{\Omega} u(x) \operatorname{div} \psi(x) d x: \psi \in C_{0}^{1}(\Omega),|\psi(x)| \leq 1, \forall x \in \Omega\right\} .
$$

The space of all functions $u \in L^{1}(\Omega)$ such that $\int_{\Omega}|\nabla u|<\infty$ is the set $B V(\Omega)$, for which $\int_{\Omega}|\nabla u|$ becomes a semi-norm.

Generalizing from the Euclidean norm to the norm given by the surface free energy function $\phi$, define the anisotropic total variation [18] as

$$
\int_{\Omega} \phi(\nabla u):=\sup \left\{\int_{\Omega} u(x) \operatorname{div} \psi(x) d x: \psi \in C_{0}^{1}(\Omega), \phi^{\circ}(\psi(x)) \leq 1, \forall x \in \Omega\right\} .
$$

Write $\operatorname{dist}(Y): \Omega \rightarrow \mathbb{R}$ for the signed distance function to a set $Y \subset \Omega$, defined by

$$
\operatorname{dist}(Y)(x):=\inf _{y \in Y}|x-y|-\inf _{y \in \Omega \backslash Y}|x-y| .
$$


2.2. Outline of the proposed methods. We give a brief outline of the two proposed methods for solving the discrete time evolution of an anisotropic mean curvature flow. For both cases, we need to solve a minimization problem to evolve the mean curvature flow by one discrete time step $h>0$. The minimization problem involves the ROF functional (1.3). There are various methods for performing this minimization. We used the split Bregman method which has the advantage that it splits the problem into two simpler problems. The first step is to solve a linear elliptic PDE. The second step is pointwise minimization, which, in some cases, has an explicit solution called shrinkage. The additional advantage is that the anisotropy only appears in the second step. In that case we are required to solve an anisotropic shrinkage problem, which we show has an explicit solution in the crystalline case. In the smooth case, no such explicit solution can be obtained. As a result, the methods used to solve this problem are very different in the two cases.

We will now give an outline of the overall method, which will be expanded on in the rest of the article.

The starting point is the isotropic case $\phi(\cdot)=|\cdot|$. The split Bregman method for the ROF functional is described in detail in Section 3.1. It involves alternatingly solving an elliptic PDE (for $u^{*}$ ) and a pointwise minimization (for $d^{*}$ ),

$$
\begin{aligned}
(1 / h-\mu \Delta) u^{*} & =f / h-\mu \nabla \cdot(d-b), \\
d^{*} & =\arg \min _{d}\|d\|_{L^{1}(\Omega)}+\frac{\mu}{2}\|d-\nabla u-b\|_{L^{2}(\Omega)}^{2},
\end{aligned}
$$

for a parameter $\mu>0$. The minimization (2.6) has an explicit solution

$$
d^{*}=\operatorname{shrink}(\nabla u+b, 1 / \mu)
$$

called shrinkage, which is defined for all $x \in \mathbb{R}^{2}, \lambda>0$, by

$$
\operatorname{shrink}(x, \lambda)= \begin{cases}\max (|x|-\lambda, 0) \frac{x}{|x|}, & \text { for } x \neq 0, \\ 0 & \text { for } x=0 .\end{cases}
$$

The application of the method to isotropic mean curvature flow is described in Section 3.2 .

For a general surface free energy function $\phi$, the elliptic PDE (2.5) remains unchanged, while the pointwise minimization step (2.6) is replaced by

$$
d^{*}=\arg \min _{d} \int_{\Omega} \phi(d)+\frac{\mu}{2}\|d-\nabla u-b\|_{L^{2}(\Omega)}^{2} .
$$

The minimization decouples at each point in $\Omega$ and thus can be written as

$$
d^{*}=\operatorname{shrink}_{\phi}(\nabla u+b, 1 / \mu),
$$

where

$$
\operatorname{shrink}_{\phi}(x, \lambda):=\arg \min _{y \in \mathbb{R}^{2}} \phi(y)+\frac{\lambda}{2}|x-y|^{2} .
$$

In the case where $\phi$ is polyhedral (see Definition (4.5)), we shall call the right hand side of (2.8) a polyhedral shrinkage problem. We obtain an explicit formula for the polyhedral shrinkage problem in Section 4.1.

For the case where $\phi$ is smooth, the method we propose is quite different. No explicit solution for (2.8) is available; instead we consider a system of nonlinear evolution equations which, in the steady state limit, solves both minimizations (2.5) and (2.6) simultaneously. The system is presented in Section 5.1, and derived in Section 5.2. 


\section{The split Bregman method}

We first lay out a known algorithm for isotropic mean curvature flow based on the split Bregman method [25]. This will be the prototype for the anisotropic mean curvature flow algorithms described later.

3.1. Overview of the split Bregman method. First, we briefly describe the split Bregman method applied to the ROF functional. For further details we refer the reader to [31].

Let $\Omega \subset \mathbb{R}^{2}$ be a bounded, connected set, $f \in B V(\Omega)$, and $\lambda>0$ be a real parameter. We seek to minimize the ROF functional

$$
\int_{\Omega}|\nabla u|+\frac{\lambda}{2}\|u-f\|_{L^{2}(\Omega)}^{2}
$$

over the set of all $u \in L^{2}(\Omega)$. The key idea is to consider the following constrained minimization problem:

$$
\min _{u, d} J(u, d)=\|d\|_{L^{1}(\Omega)}+\frac{\lambda}{2}\|u-f\|_{L^{2}(\Omega)}^{2} \quad \text { such that } \quad d=\nabla u .
$$

Then, (3.2) is solved by the Bregman iteration [4, 41]:

$$
\begin{aligned}
\left(u^{k+1}, d^{k+1}\right) & =\arg \min _{u, d} D_{J}^{p_{u}^{k}, p_{d}^{k}}\left(u, u^{k}, d, d^{k}\right)+\frac{\mu}{2}\|d-\nabla u\|_{L^{2}(\Omega)}^{2}, \\
p_{u}^{k} & :=\partial_{u} J\left(u^{k}, d^{k}\right)=\lambda\left(u^{k}-f\right), \\
p_{d}^{k} & :=\partial_{d} J\left(u^{k}, d^{k}\right)=\partial_{d}\left\|d^{k}\right\|_{L^{1}(\Omega)},
\end{aligned}
$$

where $\mu>0$ is a parameter, $\partial_{u} J(u, k)$ and $\partial_{d} J(u, k)$ denote the $u$ and $d$ subgradients of $J(u, k)$, respectively, and $D_{J}^{p_{u}^{k}, p_{d}^{k}}\left(u, u^{k}, d, d^{k}\right)$ is the Bregman distance of $J(\cdot, \cdot)$, defined by

$$
D_{J}^{p_{u}^{k}, p_{d}^{k}}\left(u, u^{k}, d, d^{k}\right):=J(u, d)+J\left(u^{k}, d^{k}\right)-\left\langle p_{u}^{k}, u-u^{k}\right\rangle-\left\langle p_{d}^{k}, d-d^{k}\right\rangle .
$$

The initial condition is set to $u^{0}=f, d^{0}=p_{u}^{0}=p_{d}^{0}=0$. Then under suitable conditions, $u^{k}$ converges to the minimizer of (3.1); see Remark 3.1. It can be shown that the iterative sequence (3.3), (3.4), and (3.5) can be equivalently written as

$$
\begin{aligned}
\left(u^{k+1}, d^{k+1}\right) & =\arg \min _{u, d}\|d\|_{L^{1}(\Omega)}+\frac{\lambda}{2}\|u-f\|_{L^{2}(\Omega)}^{2}+\frac{\mu}{2}\left\|d-\nabla u-b^{k}\right\|_{L^{2}(\Omega)}^{2}, \\
b^{k+1} & =b^{k}+\left(\nabla u^{k+1}-d^{k+1}\right),
\end{aligned}
$$

where $b^{0}=0$. The iterative method (3.6) and (3.7) is called the split Bregman method for the ROF functional (3.1). To find the minimizer of (3.6), the authors in [31] use a two-step alternating minimization:

$$
\begin{aligned}
& u^{k+1}=\arg \min _{u} \frac{\lambda}{2}\|u-f\|_{L^{2}(\Omega)}^{2}+\frac{\mu}{2}\left\|d^{k}-\nabla u-b^{k}\right\|_{L^{2}(\Omega)}^{2} \\
& d^{k+1}=\arg \min _{d}\|d\|_{L^{1}(\Omega)}+\frac{\mu}{2}\left\|d-\nabla u^{k+1}-b^{k}\right\|_{L^{2}(\Omega)}^{2} .
\end{aligned}
$$

The first minimization (3.8) can be solved via direct calculus; the Euler-Lagrange equation gives a necessary condition on the minimizing function $u^{k+1}$ :

$$
(\lambda-\mu \Delta) u^{k+1}=\lambda f-\mu \operatorname{div}\left(d^{k}-b^{k}\right),
$$


coupled with the boundary condition (see Appendix B for its derivation)

$$
\nabla u^{k+1} \cdot n=\left(d^{k}-b^{k}\right) \cdot n \quad \text { on } \partial \Omega,
$$

where $n$ is the outward normal to $\partial \Omega$.

With respect to the second minimization (3.9), as mentioned in Section 2.2, the problem decouples at every point in $\Omega$. The minimizer is described by the shrinkage operator

$$
\begin{aligned}
d^{k+1} & =\operatorname{shrink}\left(\nabla u^{k+1}+b^{k}, 1 / \mu\right) \\
& :=\max \left(\left|\nabla u^{k+1}+b^{k}\right|-1 / \mu, 0\right) \frac{\nabla u^{k+1}+b^{k}}{\left|\nabla u^{k+1}+b^{k}\right|}
\end{aligned}
$$

at each point in $\Omega$.

REMARK 3.1. The split Bregman method utilizes previously known technques in optimization. Setzer [47] proved that the split Bregman method converges to the minimizer of (3.1) by establishing that (3.6) and (3.7) is the Augmented Lagragian method (c.f. [39, Chaper 17]) and that (3.8) and (3.9) is a special case of the DouglasRachford splitting method [17]. The split Bregman method is also identical to the alternating directions method of multipliers (ADMM) [29, 30].

3.2. Isotropic mean curvature flow. We now apply the split Bregman algorithm to Chambolle's formulation for isotropic mean curvature flow.

Consider an initial closed curve $C^{0}=\partial S^{0}$, where $S^{0}$ is an open subset in $\Omega$. The goal is to approximate $C(t)$, the mean curvature flow of $C(0)=C^{0}$, after time $t>0$. For a fixed time step $h>0$, let $w_{S^{0}}^{*} \in L^{2}(\Omega)$ be the minimizer of the ROF functional (3.1) with $\lambda=1 / h$ and $f=\operatorname{dist}\left(S^{0}\right)$. Define the operator $T_{h}\left(S^{0}\right):=\left\{x \in \Omega \mid w_{S^{0}}^{*}(x)<\right.$ $0\}$. Then, for $t>0$, let

$$
S_{h}(t):=\left(T_{h}\right)^{[t / h]}\left(S^{0}\right) .
$$

It can be shown that $C_{h}(t)=\partial S_{h}(t)$ for $t>0$ is the discrete time approximation to the mean curvature flow $C(t)$ of the initial contour $C^{0}$. Furthermore, (3.12) implements a monotone curve evolution of the flow introduced by Almgren, Taylor, and Wang [1]; that is, $S \subset S^{\prime}$ implies $T_{h}(S) \subset T_{h}\left(S^{\prime}\right)$.

For the actual implementation, it is convenient to work with level set functions rather than sets. Let $u_{j}:=\operatorname{dist}\left(S^{j}\right)$, for $j=0,1,2 \ldots$ be the sequence of distance functions to the sets $S^{j}:=\left(T_{h}\right)^{j}\left(S^{0}\right)$, for $j=0,1,2 \ldots$ Note that the zero level sets of $u_{j}(x)$ form the discrete time approximation of the evolution of $C^{0}$.

We outline the split Bregman approach to isotropic mean curvature flow in Algorithm 1. The loops in $n$ and $m$ are called the outer loop and the inner loop, respectively. The inner loop iteratively solves (3.8) and (3.9) to minimize (3.6). The outer loop iteratively performs (3.6) and (3.7). The constants $N$ and $M$ may be chosen manually, or alternatively the outer and inner loops may be carried out until consecutive iterates are within a given tolerance. The loop in $j$ corresponds to the discrete time iteration of the approximation $C(j h) \approx C_{h}(j h)=\left\{u_{j}(x)=0\right\}$.

\section{Crystalline mean curvature flow}

To incorporate the anisotropy of $\phi$ in the split Bregman formulation of mean curvature flow, one must consider the anisotropic ROF problem:

$$
\int_{\Omega} \phi(\nabla u)+\frac{\lambda}{2}\|u-f\|_{L^{2}(\Omega)}^{2} .
$$




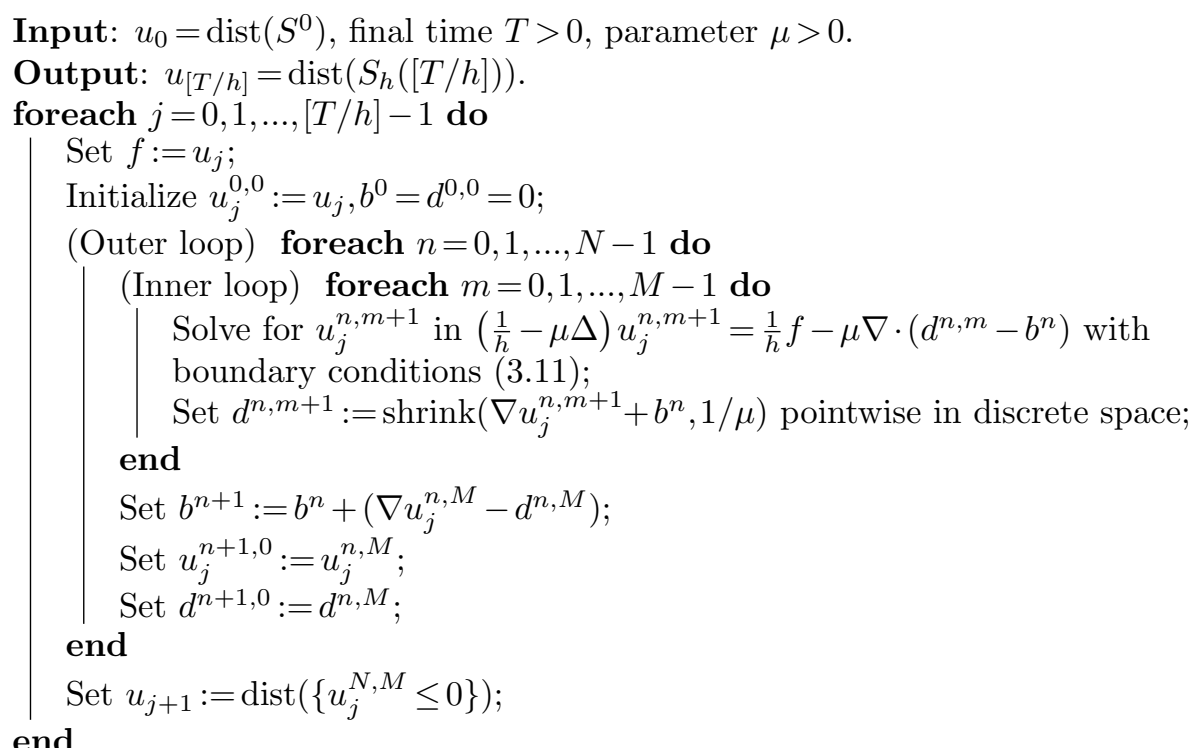

Algorithm 1: Isotropic mean curvature flow using the split Bregman algorithm.

Following the derivation in Section 3.1, the anisotropy appears only in the pointwise minimization (3.9). This leads us to consider the anisotropic shrinkage problem (2.7).

4.1. Polyhedral shrinkage. In this section we show that the generalized shrinkage problem is equivalent to the projection onto the Wulff shape. Subsequently, we obtain an explicit solution formula for the generalized shrinkage problem for a polyhedral norm (see (4.5) for a definition).

We start by writing $\phi$ as in (2.2),

$$
\phi(x)=\sup _{z \in \mathcal{W}_{\phi}} z \cdot x
$$

and define $\pi_{\mathcal{W}_{\phi}}(z)$ to be the projection of $z$ onto $\mathcal{W}_{\phi}$,

$$
\pi_{\mathcal{W}_{\phi}}(y):=\arg \min _{x \in \mathcal{W}_{\phi}}|x-y|^{2}
$$

Consider the minimization problem

$$
x^{*}=x^{*}(y):=\arg \min _{x \in \mathbb{R}^{n}}\left\{\phi(x)+\frac{1}{2}|x-y|^{2}\right\} .
$$

REMARK 4.1. Note the relation of $x^{*}(y)$ to the generalized shrinkage function introduced in Section 2.2:

$$
x^{*}(y)=\operatorname{shrink}_{\phi}(y, 1) .
$$

The anisotropic shrinkage $\operatorname{shrink}_{\phi}(y, \lambda)$ for arbitrary $\lambda>0$ is equivalent to $x^{*}(y)$ if $\phi$ in (4.3) is scaled by $1 / \lambda$.

We claim the following characterization holds. 
Lemma 4.1. Denote $x^{*}(y)$ as the minimizer of (4.3). Then

$$
x^{*}(y)=y-\pi_{\mathcal{W}_{\phi}}(y) .
$$

Proof. Rewrite the minimization in (4.3) using (4.2) as

$$
\min _{x \in \mathbb{R}^{n}} \max _{z \in \mathcal{W}_{\phi}}\left\{\frac{1}{2}|x-y|^{2}+z \cdot x\right\} .
$$

Since the inner function is convex in $x$ and affine in $y$, we can interchange the order of min and max to obtain

$$
\max _{z \in \mathcal{W}_{\phi}} \min _{x \in \mathbb{R}^{n}}\left\{\frac{1}{2}|x-y|^{2}+z \cdot x\right\} .
$$

Evaluate the inner problem to obtain

$$
x^{*}(y)=y-z,
$$

which results in $\max _{z \in \mathcal{W}_{\phi}}\left\{-\frac{1}{2} z^{2}+z \cdot y\right\}$, or equivalently,

$$
\min _{z \in \mathcal{W}_{\phi}}\left\{\frac{1}{2}|z-y|^{2}\right\}
$$

The last problem is a projection problem, the minimizer given by

$$
z=\pi_{\mathcal{W}_{\phi}}(y) .
$$

The last result, together with $x^{*}(y)=y-z$, yields (4.4).

Proposition 4.2. $y \in \mathcal{W}_{\phi}$ if and only if $x^{*}(y)=0$.

Proof. This is an immediate consequence of (4.4).

From hereon in this section, we let $\phi$ be a polyhedral norm: given a set of normals $\mathcal{N}=\left\{n_{i}\right\}_{i=1}^{k} \subset \mathbb{R}^{2}$ ordered clockwise (define $n_{k+1}=n_{1}$ and $n_{0}=n_{k}$ ), write

$$
\phi(x)=\max _{n_{i} \in \mathcal{N}} n_{i} \cdot x .
$$

Partition $\mathbb{R}^{2} \backslash\{0\}$ into the regions

$$
\begin{aligned}
C_{i} & =\left\{x \neq 0 \mid n_{i} \cdot x>n_{j} \cdot x \text { for } j=1, \ldots, k\right\} \quad i=1, \ldots, k, \\
R_{i, i+1} & =\left\{x \neq 0 \mid n_{i} \cdot x=n_{i+1} \cdot x>0\right\} \quad i=1, \ldots, k .
\end{aligned}
$$

Thus, $\phi$ is smooth in $C_{i}$ and has a kink along the rays $R_{i, i+1}$ for each $i=1, \ldots, k$. In light of Proposition 4.2, we are left with characterizing the minimizer for the case $y \notin \mathcal{W}_{\phi}$ (equivalently $\phi^{\circ}(y)>1$ ).

Theorem 4.3 (Polyhedral Shrinkage Formula). Assume that the normals in (4.5) are ordered clockwise, and that $\phi^{\circ}(y)>1$. Define, for each $i=1, \ldots, k$,

$$
\lambda_{i}=\lambda_{i}(y):=\frac{\left(n_{i}-n_{i+1}\right) \cdot y+\left\|n_{i+1}\right\|^{2}-n_{i} \cdot n_{i+1}}{\left\|n_{i}-n_{i+1}\right\|^{2}} .
$$


Then, there exists $j \in\{1, \ldots, k\}$ such that either

$$
\lambda_{j}(y) \in[0,1]
$$

or

$$
\lambda_{j}(y)>1 \text { and } \lambda_{j-1}(y)<0 .
$$

Furthermore, in the former case,

$$
x^{*}(y)=y-\left(n_{j} \lambda_{j}(y)+n_{j+1}\left(1-\lambda_{j}(y)\right)\right),
$$

provided $x^{*}(y) \cdot\left(n_{j} \lambda_{j}(y)+n_{j+1}\left(1-\lambda_{j}(y)\right)\right) \geq 0$, and for the latter case,

$$
x^{*}(y)=y-n_{j} .
$$

Proof. Start by taking the subdifferential of (4.3),

$$
0 \in \partial \phi\left(x^{*}\right)+\left(x^{*}-y\right), \quad x^{*}=x^{*}(y) .
$$

The subdifferential $\partial \phi$ can be explicitly solved:

$$
\partial \phi(x)=\left\{\begin{array}{lll}
n_{j} & \text { if } & x \in C_{j} \text { for some } j \in\{1, \ldots, k\}, \\
\operatorname{conv}\left\{n_{j}, n_{j+1}\right\} & \text { if } \quad x \in R_{j, j+1} \text { for some } j \in\{1, \ldots, k\} .
\end{array}\right.
$$

By Proposition 4.2, $y \notin \mathcal{W}_{\phi}$ implies that $x^{*}(y) \neq 0$. Since $\left\{C_{i}, R_{i, i+1}\right\}_{i=1}^{k}$ partitions $\mathbb{R}^{2} /\{0\}$, it will be sufficient to consider the two cases: either $x^{*}(y) \in C_{j}$ or $x^{*}(y) \in$ $R_{j, j+1}$, for some $j \in\{1, \ldots, k\}$.

Rearrange (4.10) and consider the case when $x^{*}(y) \in R_{j, j+1}$ for some $j \in\{1, \ldots, k\}$. Then we have

$$
y-\left(n_{j} \lambda+n_{j+1}(1-\lambda)\right)=x^{*}(y)
$$

for some unique $\lambda \in[0,1]$. Muliplying (4.12) by $n_{j}$ and $n_{j+1}$ separately, and noting that $n_{j} \cdot x^{*}(y)=n_{j+1} \cdot x^{*}(y)$, one can solve for $\lambda$ : the solution is $\lambda=\lambda_{j}(y)$ as per (4.6). Setting $\lambda=\lambda_{j}(y)$ in (4.12) gives (4.9). However, since there may be more than one $\lambda_{j}$ such that (4.7) holds, we must ensure that the correct $j$ was chosen. Thus, one needs to check that $x^{*}(y)$ actually lies on $R_{j, j+1}$; this is achieved by enforcing $x^{*}(y) \cdot\left(n_{j} \lambda_{j}(y)+n_{j+1}\left(1-\lambda_{j}(y)\right)\right) \geq 0$.

For the case $x^{*}(y) \in C_{j}$ for some $j \in\{1, \ldots, k\}$, we have

$$
y-n_{j}=x^{*}(y) .
$$

To see that $\lambda_{j}(y)>1$ and $\lambda_{j-1}(y)<0$ in this case, note that if $x^{*}(y) \in C_{j}$,

$$
n_{j} \cdot x^{*}(y)>n_{i} \cdot x^{*}(y), \quad \text { for all } i \neq j .
$$

Since $y=x^{*}(y)+n_{j}$, formula (4.6) with the former inequalities imply

$$
\begin{aligned}
\lambda_{j}(y) & =\frac{\left(n_{j}-n_{j+1}\right) \cdot x^{*}(y)}{\left\|n_{j}-n_{j+1}\right\|^{2}}+1>1, \\
\lambda_{j-1}(y) & =\frac{\left(n_{j-1}-n_{j}\right) \cdot x^{*}(y)}{\left\|n_{j}-n_{j+1}\right\|^{2}}<0 .
\end{aligned}
$$




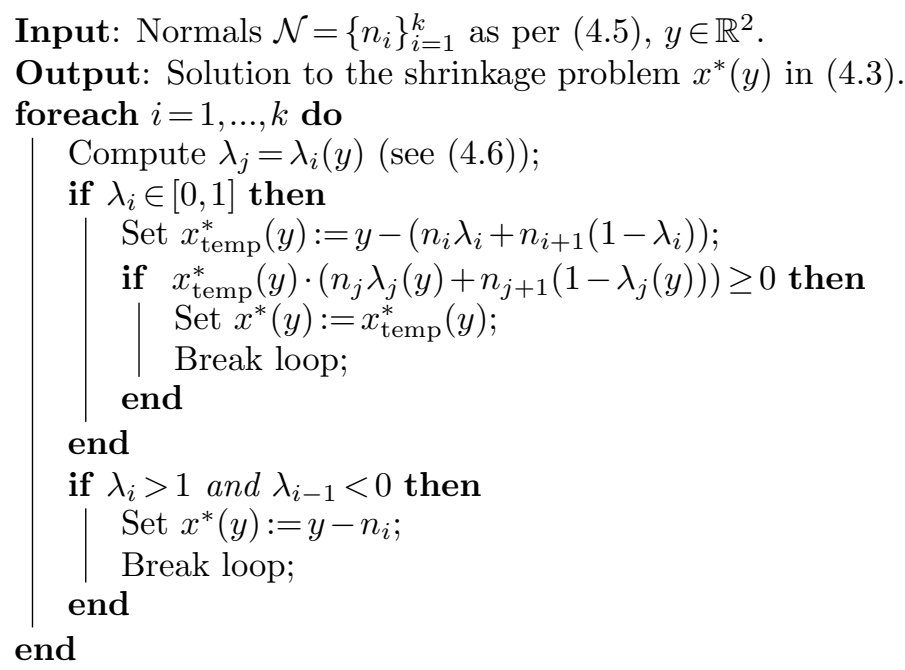

Algorithm 2: Polyhedral shrinkage algorithm.

Theorem 4.3 can be immediately restated as an algorithm for the polyhedral shrinkage problem; see Algorithm 2.

We illustrate the result of the Proposition 4.2 in Figure 4.1 by computing shrinkage for the "octagon norm"

$$
\phi_{8}(x)=\max _{n_{i} \in \mathcal{N}_{8}} n_{i} \cdot x
$$

where the eight normals are $\mathcal{N}_{8}=\{( \pm 1,0),(0, \pm 1),( \pm 1, \pm 1) / \sqrt{2},( \pm 1, \mp 1) / \sqrt{2}\}$.

4.2. Algorithm for the crystalline mean curvature flow. The polyhedral shrinkage algorithm (Algorithm 2) provides the key ingredient to modify Algorithm 1 for crystalline $\phi$. We omit presenting the complete algorithm for the crystalline case since it only differs from Algorithm 1 at a single line; namely, within the inner loop, the line "Set $d^{n, m+1}:=\operatorname{shrink}\left(\nabla u_{j}^{n, m+1}-b^{n}, 1 / \mu\right)$ pointwise in discrete space" should be replaced by Algorithm 2 with inputs $y=\nabla u_{j}^{n, m+1}-b^{n}$ and the set of normals $\left\{n_{i}\right\}_{i=1}^{k}$ corresponding to the Frank diagram, scaled accordingly by $1 / \mu$; see Remark 4.1.

Remark 4.2. As pointed out in [9], the necessary modification to Algorithm 1 for solving (1.6) with mobility function $\beta(x)=\phi(x) /|x|$ is the last line which should be replaced by:

$$
\text { Set } u_{j+1}:=\operatorname{dist}_{\phi^{\circ}}\left(\left\{u_{j}^{N, M} \leq 0\right\}\right),
$$

where

$$
\operatorname{dist}_{\phi^{\circ}}(Y)(x):=\inf _{y \in Y} \phi^{\circ}(x-y)-\inf _{y \in \Omega \backslash Y} \phi^{\circ}(x-y)
$$

is the signed distance function to a set in the $\phi^{\circ}$ metric.

\section{Smooth anisotropic mean curvature flow}

We saw in the last section that an explicit characterization of shrinkage was possible for a crystalline (or polyhedral) anisotropy on $\phi$. However, for smooth anisotropic 

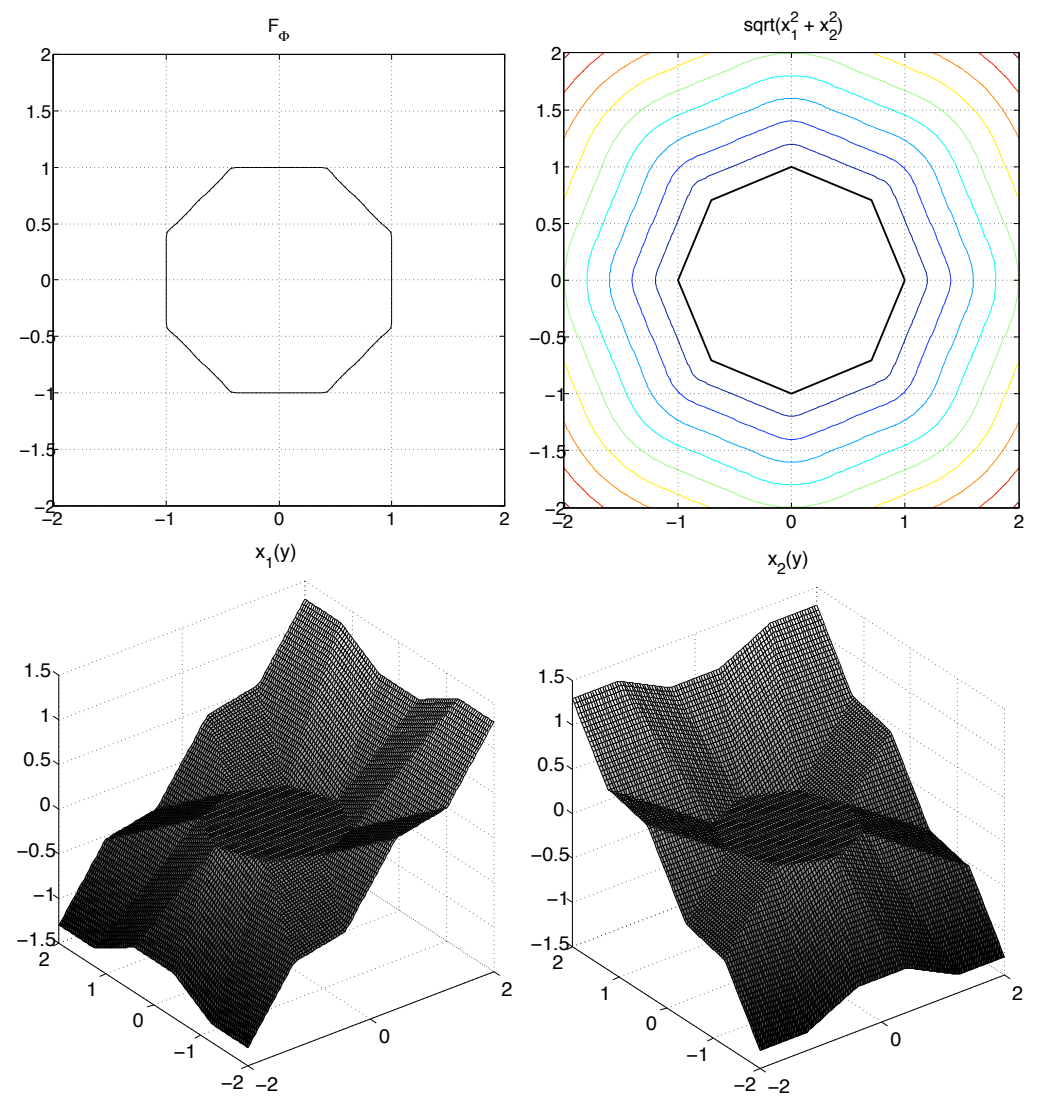

Fig. 4.1: Top left: the Frank diagram for the "octagon norm" $\phi_{8}$. Top right: contour plot of $\left|x^{*}(y)\right|$. The dark contour is the zero level set; note that this is the Wulff shape for $\phi_{8}$. Bottom left and right: a surface plot of the components of $x^{*}(y)=$ $\left(x_{1}(y), x_{2}(y)\right)$.

$\phi$, an explicit formula is not available in general. We give an example to illustrate why this formula is not available below.

Instead, the shrinkage formula needs to be computed numerically. One option is to implement an approximation scheme to project onto a smooth unit ball of the dual norm, say by a Newton's method approach, or by means of an efficient distance function solver such as the algorithm proposed in [50]. Due to the convexity of the unit ball, Newton's method is expected to converge quickly and thus provide accurate and efficient computational solutions to the shrinkage problem. One may also resort to a general Legendre transform solver [36]. In both of the references cited, the computed solution is on a uniform mesh, so interpolation or extrapolation is required.

We shall take a different route, which does not involve any explicit optimization problems. Our approach, motivated by [3], is to derive an inverse scale space flow arising from the split Bregman method. This approach is slow: each discrete time step of the mean curvature flow is slower than simply solving the equation by the standard level set method, which is the benchmark for speed. While the implementation is not efficient, it complements the method in the crystalline case. We formally derive a 
nonlinear system of PDE's (5.2) which solves for the minimizer of anisotropic ROF functional (4.1) in the steady state limit. In Section 5.3, we show that this nonlinear flow posesses several interesting properties; in particular, it solves a semi-implicit Euler scheme for the mean curvature flow PDE (1.5).

To illustrate the difficulty of shrinkage for smooth anisotropies, consider the smooth norm

$$
\phi(x)=\sqrt{2 x_{1}^{2}+x_{2}^{2}}, \quad x=\left(x_{1}, x_{2}\right)
$$

Its dual norm $\phi^{\circ}(x)=\sqrt{\frac{1}{2} x_{1}^{2}+x_{2}^{2}}$, has a unit ball of an ellipse elongated in the $x_{1}$ direction. Thus, shrinkage for $\phi$ amounts to computing the closest point to this ellipse. A calculus argument shows that such a task requires one to solve a non-trivial quartic equation. One may easily see that deriving a shrinkage formula for a more complicated smooth norm becomes quickly difficult.

5.1. Presentation of the inverse scale space equations. We begin by presenting the system of PDE's describing the inverse scale space flow in the case of smooth anisotropy. This system arises from (3.3), (3.4), and (3.5), and will be derived in Section 5.2. Throughout this section, we shall decompose the norm $\phi$ as follows:

$$
\phi(x)=\gamma(\omega)|x|, \quad \omega=\tan ^{-1}\left(x_{2} / x_{1}\right) .
$$

The function $\gamma$ of phase is $2 \pi$-periodic and 0-homogeneous in $x$. Furthermore, we shall assume that $\phi$ and $\gamma$ are smooth functions.

The evolution is in the time parameter $s$, independent of $x$. We suppress the parameter $x$ in the notation and write $u=u(s), \theta=\theta(s)$. The inverse scale space equations consist of two differential equations:

$$
\begin{array}{lrl}
\frac{\partial \theta}{\partial s}=\frac{p^{\perp} \cdot \nabla u}{\gamma(\theta)+\gamma^{\prime \prime}(\theta)}, & p=(\cos \theta, \sin \theta), \\
\frac{\partial u}{\partial s}=h(\Delta u-\nabla \cdot d), & d=(p \cdot \nabla u) p .
\end{array}
$$

The initial conditions are

$$
\begin{aligned}
& \theta(0)=\tan ^{-1}\left(-f_{x} / f_{y}\right), \\
& u(0)=f .
\end{aligned}
$$

5.2. Formal derivation of the inverse scale space equations. In this section, we formally derive the inverse scale space Equation (5.2) by taking the EulerLagrange equations of the split Bregman functional (3.3).

Consider the Bregman iterative update formulas (3.3), (3.4), and (3.5) for the split Bregman minimization problem (3.2). For clarity, we write out the right hand side of (3.3) completely using the definition of the Bregman distance:

$$
\min _{d, u} \int \gamma(\theta)|d|+\frac{1}{2 h} \int|u-f|^{2}-\left\langle p_{u}^{k}, u-u^{k}\right\rangle-\left\langle p_{d}^{k}, d-d^{k}\right\rangle+\frac{\mu}{2} \int|d-\nabla u|^{2},
$$

where $\theta=\theta(d)=\tan ^{-1}\left(d_{2} / d_{1}\right)$, and $d=\left(d_{1}, d_{2}\right)$. Recall the subgradients:

$$
\begin{aligned}
& p_{d}^{k}=\partial_{d^{k}} \int \gamma\left(\theta\left(d^{k}\right)\right)\left|d^{k}\right|, \\
& p_{u}^{k}=\frac{1}{h}\left(u^{k}-f\right), \quad \text { since } \lambda=1 / h .
\end{aligned}
$$


First, taking the Euler-Lagrange derivative of the functional with respect to $d$ gives

$$
p_{d}^{k+1}-p_{d}^{k}+\mu\left(d^{k}-\nabla u^{k}\right)=0 .
$$

Observing (5.7) as a forward Euler update scheme over a time variable $s$ with step size $\mu$, it approximates the PDE

$$
\frac{\partial p_{d}}{\partial s}=\nabla u-d
$$

For notational simplicity, let us denote $d=R p$, for $R$ a scalar and $p=(\cos \theta, \sin \theta)$ a unit vector. By the smoothness assumption of $\gamma$, for $d \neq 0$,

$$
\begin{aligned}
p_{d}=\partial_{d} \int \gamma(\theta)|d| & =\frac{d}{|d|} \gamma(\theta)+|d| \gamma^{\prime}(\theta) \nabla_{d} \theta(d) \\
& =\frac{d}{|d|} \gamma(\theta)+\frac{d^{\perp}}{|d|} \gamma^{\prime}(\theta) \quad \text { where } d^{\perp}=\left(-d_{2}, d_{1}\right) \\
& =p \gamma(\theta)+p^{\perp} \gamma^{\prime}(\theta) \quad \text { where } p^{\perp}=\left(-p_{2}, p_{1}\right),
\end{aligned}
$$

thus

$$
\begin{aligned}
\frac{\partial p_{d}}{\partial s}= & (-\sin \theta, \cos \theta) \frac{\partial \theta}{\partial s} \gamma(\theta)+(\cos \theta, \sin \theta) \frac{\partial \theta}{\partial s} \gamma^{\prime}(\theta) \\
& \quad-(\cos \theta, \sin \theta) \frac{\partial \theta}{\partial s} \gamma^{\prime}(\theta)+(-\sin \theta, \cos \theta) \frac{\partial \theta}{\partial s} \gamma^{\prime \prime}(\theta) \\
= & p^{\perp}\left(\gamma(\theta)+\gamma^{\prime \prime}(\theta)\right) \frac{\partial \theta}{\partial s}
\end{aligned}
$$

Substituting the latter result into (5.8),

$$
p^{\perp}\left(\gamma(\theta)+\gamma^{\prime \prime}(\theta)\right) \frac{\partial \theta}{\partial s}=\nabla u-R p
$$

If we multiply (5.9) by $p$,

$$
\begin{aligned}
0 & =p \cdot \nabla u-R|p|^{2} \\
R & =p \cdot \nabla u .
\end{aligned}
$$

If we multiply (5.9) by $p^{\perp}$,

$$
\begin{aligned}
\left(\gamma(\theta)+\gamma^{\prime \prime}(\theta)\right) \frac{\partial \theta}{\partial s} & =p^{\perp} \cdot \nabla u-R p^{\perp} \cdot p, \\
\frac{\partial \theta}{\partial s} & =\frac{p^{\perp} \cdot \nabla u}{\gamma(\theta)+\gamma^{\prime \prime}(\theta)} .
\end{aligned}
$$

This is the first differential equation in (5.2).

Next take the derivative of the functional in (5.4) with respect to $u$ :

$$
p_{u}^{k+1}-p_{u}^{k}-\mu\left(\operatorname{div} d^{k}-\Delta u^{k}\right)=0 .
$$

Since the $u$ subgradient is explicitly $p_{u}^{k}=\frac{1}{h}\left(u^{k}-f\right)$,

$$
\frac{1}{h}\left(u^{k+1}-f\right)-\frac{1}{h}\left(u^{k}-f\right)-\mu\left(\operatorname{div} d^{k}-\Delta u^{k}\right)=0 .
$$


Again taking $\mu$ to be the step size of a forward Euler discretization, the PDE for $u$ becomes

$$
\frac{\partial u}{\partial s}=h(\Delta u-\nabla \cdot d)
$$

This is the second differential equation in (5.2).

Finally, we derive the initial conditions (5.3). Following [3], the initial conditions for an inverse scale space flow are $d(0)=0, p_{d}(0)=0, p_{u}(0)=0$. Immediately, we have $R(0)=|d(0)|=0$. Since $0=p_{u}(0)=(u(0)-f)$, we also have $u(0)=f$. Consequently, since $0=R(0)=p(0) \cdot \nabla u(0)=p(0) \cdot \nabla f$ holds, $p(0)=\left(-f_{y}, f_{x}\right) \equiv \nabla^{\perp} f$, and therefore, $\theta(0)=\tan ^{-1}\left(-f_{x}, f_{y}\right)$.

5.3. Properties of the inverse scale space flow. Next we present analytical properties of the inverse scale space flow (5.2). For what follows, we use the notation from Section 5.2:

$$
\begin{aligned}
p(s) & =(\cos \theta(s), \sin \theta(s)), \\
R(s) & =p(s) \cdot \nabla u(s) .
\end{aligned}
$$

We prove two main results. The first concerns convergence of $\nabla u(s)$ to $d(s)$ as $s \rightarrow \infty$. It gives a convergence proof provided that $R(s)$ stays positive in the limit. In general, we have observed that $R(s)$ may be zero for some $s>0$. For sufficiently smooth convex $f$, however, $R(s)$ appears to stay positive for all $s>0$.

Proposition 5.1. Assume $u, d, \theta, R$, and $p$ are sufficiently smooth solutions of (5.2) and (5.3). Suppose there exists $s^{*}>0$ such that

$$
R(s) \geq R_{0}>0, \quad \text { for all } s>s^{*} .
$$

Then, $\|\nabla u(s)-d(s)\|_{L^{2}(\Omega)} \rightarrow 0$ as $s \rightarrow \infty$.

The second result establishes a connection between the variational formulation and the mean curvature PDE (1.8).

Proposition 5.2. Assume the hypotheses of Proposition 5.1. Further assume that $u, d$, and $\theta$ have sufficiently smooth limits as $s \rightarrow \infty$, namely $u_{\infty}, d_{\infty}$, and $\theta_{\infty}$, respectively, and $\left|\nabla u_{\infty}\right| \neq 0$. Then

$$
\frac{u_{\infty}-f}{h}=\left(\gamma\left(\theta_{\infty}\right)+\gamma^{\prime \prime}\left(\theta_{\infty}\right)\right) \operatorname{div} \frac{\nabla u_{\infty}}{\left|\nabla u_{\infty}\right|}|\nabla f| .
$$

Remark 5.1. Proposition 5.2 is a consistency result that recovers the level set PDE for mean curvature flow (1.8) from the inverse scale space equations (5.2). That is, solving the inverse scale space equation to steady state is equivalent to a semi-implicit forward Euler scheme for the time dependent PDE (1.8). While explicit forward Euler schemes for elliptic PDE's suffer time step restrictions $h=O\left(d x^{2}\right)$ (where $d x$ is the spacial discretization), the latter property of the inverse scale space flow suggests that it may be free from such restrictions. Note that (5.13) is a different semi-implicit Euler scheme from (1.9) of [15] since the curvature term $\operatorname{div}(\nabla u /|\nabla u|)$ in the former is fully implicit.

We begin by proving several immediate consequences of (5.2) and (5.3): 
Lemma 5.3. Assume the hypotheses of Proposition 5.1. Then,

1. $u(s)=h\left(\gamma(\theta(s))+\gamma^{\prime \prime}(\theta(s))\right) \operatorname{div} p(s)+f$, for all $s \geq 0$,

2. $\frac{\partial}{\partial s}\|u(s)\|_{L^{2}(\Omega)}^{2} \leq 0$ (decrease in $L^{2}$ energy),

3. If $R(s) \geq 0$ then $\frac{\partial}{\partial s}\|\nabla u(s)-d(s)\|_{L^{2}(\Omega)} \leq 0$,

4. $\frac{\partial \theta}{\partial s}(s)>0$ for $s \geq 0$ until steady state. (d turns counter-clockwise)

Further assume $u, d$, and $\theta$ have sufficiently smooth limits as $s \rightarrow \infty$, namely $u_{\infty}$, $d_{\infty}$, and $\theta_{\infty}$, respectively. Then,

5. $\nabla u_{\infty}=d_{\infty}$,

6. $\frac{\partial}{\partial s} D\left(u_{\infty}, u(s), d_{\infty}, d(s)\right) \leq 0$. (decay of Bregman distance)

Proof. For simplicity of notation, we suppress the inverse scale space parameter $s$ in the proofs.

1. One can verify directly: from the derivation in Section $5.2, \operatorname{div} p_{d}=\operatorname{div}(p \gamma+$ $\left.p^{\perp} \gamma\right)=\left(\gamma+\gamma^{\prime \prime}\right) \operatorname{div} p$. By (5.2), (5.8), the latter result, and interchanging $\nabla$ and $\partial / \partial s$,

$$
\frac{\partial u}{\partial s}=h \operatorname{div}(\nabla u-d)=h \operatorname{div} \frac{\partial p_{d}}{\partial s}=h \frac{\partial}{\partial s}\left(\gamma+\gamma^{\prime \prime}\right) \operatorname{div} p .
$$

Integrating over $s$, coupled by the initial conditions $u(0)=f, p(0)=\nabla^{\perp} f$, one arrives at the desired result.

2. By direct differentiation,

$$
\begin{aligned}
\frac{1}{2} \frac{\partial}{\partial s}\|u\|_{L^{2}(\Omega)}^{2} & =\int_{\Omega} u \frac{\partial u}{\partial s} d x \\
& =h \int_{\Omega} u(\Delta u-\operatorname{div} d) \\
& =h\left(-\int_{\Omega}|\nabla u|^{2}+\int_{\Omega} \nabla u \cdot d\right) \\
& =h\left(-\|\nabla u\|_{L^{2}(\Omega)}^{2}+\|R\|_{L^{2}(\Omega)}^{2}\right) \\
& \leq 0 .
\end{aligned}
$$

The last inequality holds since by (5.11), $|R|=|p \cdot \nabla u| \leq|\nabla u|$.

3. Assume $\gamma+\gamma^{\prime \prime}>0$ for well-posedness and $R(s) \geq 0$. Then we can define a seminorm

$$
\|\cdot\|_{\left(\gamma+\gamma^{\prime \prime}\right) R}:=\left\langle\left(\gamma(\theta)+\gamma^{\prime \prime}(\theta)\right) R(s) \cdot, \cdot\right\rangle^{1 / 2},
$$

where $\langle\cdot, \cdot\rangle$ is the $L^{2}$ inner product in $\Omega$. Since,

$$
\frac{\partial d}{\partial s}=\frac{\partial R}{\partial s} p+R \frac{\partial p}{\partial s}=\frac{\partial R}{\partial s} p+p^{\perp} \frac{\partial \theta}{\partial s}
$$


we have

$$
\begin{aligned}
\frac{\partial}{\partial s} \frac{1}{2}\|\nabla u-d\|_{L^{2}(\Omega)}^{2} & =\left\langle\nabla u-d, \frac{\partial}{\partial s}(\nabla u-d)\right\rangle \\
& =-\left\langle\Delta u-\operatorname{div} d, \frac{\partial u}{\partial s}\right\rangle-\left\langle\nabla u-d, \frac{\partial R}{\partial s} p+R p^{\perp} \frac{\partial \theta}{\partial s}\right\rangle \\
& =-\frac{1}{h}\left\|\frac{\partial u}{\partial s}\right\|_{L^{2}(\Omega)}^{2}-\left\langle p \cdot(\nabla u-d), \frac{\partial R}{\partial s}\right\rangle-\left\langle p^{\perp} \cdot(\nabla u-d), R \frac{\partial \theta}{\partial s}\right\rangle \\
& =-\frac{1}{h}\left\|\frac{\partial u}{\partial s}\right\|_{L^{2}(\Omega)}^{2}-\left\langle R-R, \frac{\partial R}{\partial s}\right\rangle-\left\langle\left(\gamma(\theta)+\gamma^{\prime \prime}(\theta)\right) \frac{\partial \theta}{\partial s}, R \frac{\partial \theta}{\partial t s}\right\rangle \\
& =-\frac{1}{h}\left\|\frac{\partial u}{\partial s}\right\|_{L^{2}(\Omega)}^{2}-\left\|\frac{\partial \theta}{\partial s}\right\|_{\left(\gamma+\gamma^{\prime \prime}\right) R}^{2} \\
& \leq 0 .
\end{aligned}
$$

4. Clearly, $\frac{\partial \theta}{\partial s}(0)=\left|\nabla^{\perp} f\right|^{2} /\left(\gamma(\theta(0))+\gamma^{\prime \prime}(\theta(0))\right)>0$. Consider the first instance $s=T$ for which $\frac{\partial \theta}{\partial s}(T)=0$. Then by $(5.2), p(T) \cdot \nabla^{\perp} u(T)=0$, thus $p(T)=$ $\pm \nabla u(T) /|\nabla u(T)|$. Also, by (5.2), $R(T)= \pm|\nabla u|$. Therefore, $d(T)=\nabla u(T)$, and so $\frac{\partial u}{\partial s}(T)=h \operatorname{div}(d(T)-\nabla u(T))=0$, i.e. the flow has reached a steady state at $s=T$.

5. Suppose $\nabla u_{\infty}-d_{\infty}=v$, for some constant $v \in \mathbb{R}^{2}$. Based on the notation (5.10) and (5.11), denote $p_{\infty}=\left(\cos \theta_{\infty}, \sin \theta_{\infty}\right)$ and $R_{\infty}=p_{\infty} \cdot \nabla u_{\infty}$. We prove $v=0$ by showing that $p_{\infty} \cdot v=p_{\infty}^{\perp} \cdot v=0$. First,

$$
\begin{aligned}
p_{\infty} \cdot v & =p_{\infty} \cdot \nabla u_{\infty}-p_{\infty} \cdot d_{\infty} \\
& =R_{\infty}-R_{\infty}=0 .
\end{aligned}
$$

Next, since $\frac{\partial \theta_{\infty}}{\partial s}=0$,

$$
\begin{aligned}
p_{\infty}^{\perp} \cdot v & =p_{\infty}^{\perp} \cdot \nabla u_{\infty}-p_{\infty}^{\perp} \cdot d_{\infty} \\
& =\left(\gamma\left(\theta_{\infty}\right)+\gamma^{\prime \prime}\left(\theta_{\infty}\right)\right) \frac{\partial \theta_{\infty}}{\partial s}-p_{\infty}^{\perp} \cdot R_{\infty} p_{\infty} \\
& =0-0=0 .
\end{aligned}
$$

Therefore, $v=0$.

6. By direct differentiation:

$$
\begin{aligned}
\frac{\partial}{\partial s} D\left(u_{\infty}, u, d_{\infty}, d\right)= & \left\langle p_{d}, \frac{\partial d}{\partial s}\right\rangle+\left\langle\frac{u-f}{h}, \frac{\partial u}{\partial s}\right\rangle-\left\langle\frac{1}{h} \frac{\partial u}{\partial s}, u_{\infty}-u\right\rangle \\
& -\left\langle\frac{u-f}{h}, \frac{\partial u}{\partial s}\right\rangle-\left\langle\frac{\partial p_{d}}{\partial s}, d_{\infty}-d\right\rangle-\left\langle p_{d}, \frac{\partial d}{\partial s}\right\rangle \\
= & -\left\langle\operatorname{div}(\nabla u-d), u_{\infty}-u\right\rangle-\left\langle\nabla u-d, d_{\infty}-d\right\rangle \\
= & \left\langle\nabla u-d, \nabla u_{\infty}-\nabla u-\left(d_{\infty}-d\right)\right\rangle \\
= & -\|\nabla u-d\|_{L^{2}(\Omega)}^{2} \quad \text { since } \nabla u_{\infty}=d_{\infty} \\
\leq & 0 .
\end{aligned}
$$


Result 4 of Lemma 5.3 shows that the direction of $d(s)$ is counter-clockwise as $s$ increases. Initially, $d(0)$ is perpendicular to $\nabla u(0)=\nabla f$. Thus, $d(t)$ turns counterclockwise until it coincides with $\nabla u$, at which point $\frac{\partial \theta}{\partial s}=0$ and steady state is reached. Note that $\nabla u$ may also "turn", but does so at a much slower rate, scaled by $h$.

Result 5 of Lemma 5.3 hinges on the assumption that both $u$ and $d$ converge to steady state solutions. While Proposition 5.1 gives a convergence proof under certain conditions, whether such assumption is generally true is still an open question, although all of our numerical tests confirmed the affirmative.

We close this section by proving the two main propositions.

Proof. [Proof of Proposition 5.1] Again, for simplicity of notation, we suppress the inverse scale space parameter $s$. We may assume that there exists $B>0$ such that,

$$
B \geq \gamma(\theta)+\gamma^{\prime \prime}(\theta)>0
$$

By (5.9), we can write

$$
\|\nabla u-d\|_{L^{2}(\Omega)}^{2}=\left\|\left(\gamma(\theta)+\gamma^{\prime \prime}(\theta)\right) \frac{\partial \theta}{\partial s}\right\|_{L^{2}(\Omega)}^{2} .
$$

From (5.14) (the steps therein), (5.12), and the last equality, for $s>s^{*}$,

$$
\begin{aligned}
\frac{\partial}{\partial s} \frac{1}{2}\|\nabla u-d\|_{L^{2}(\Omega)}^{2} & \leq-\left\langle\frac{\partial \theta}{\partial s}, \frac{\partial \theta}{\partial s}\left(\gamma(\theta)+\gamma^{\prime \prime}(\theta)\right) R(s)\right\rangle \\
& \leq-\frac{R_{0}}{B}\left\langle\frac{\partial \theta}{\partial s}, \frac{\partial \theta}{\partial s}\left(\gamma(\theta)+\gamma^{\prime \prime}(\theta)\right)^{2}\right\rangle \\
& =-\frac{R_{0}}{B}\|\nabla u-d\|_{L^{2}(\Omega)}^{2} .
\end{aligned}
$$

By Gronwall's inequality, $\quad\|\nabla u(s)-d(s)\|_{L^{2}(\Omega)} \leq\|\nabla u(0)-d(0)\|_{L^{2}(\Omega)} e^{-\frac{2 R_{0}}{B} s}=$ $\|\nabla f\|_{L^{2}(\Omega)} e^{-\frac{2 R_{0}}{B} s}$; thus taking $s \rightarrow \infty$ we have our result.

Proof. [Proof of Proposition 5.2] Since $\nabla u_{\infty}=d_{\infty}$ (result 5 of Lemma 5.3) and $p_{\infty}$ is the unit vector pointing in the direction of $d_{\infty}$, we have

$$
p_{\infty}=\frac{\nabla u_{\infty}}{\left|\nabla u_{\infty}\right|} .
$$

Substituting the above into result 1 of Lemma 5.3 and rearranging gives the desired result. Note that $|\nabla f|=1$, as $f$ is a signed distance function.

5.4. Inverse scale space algorithm for smooth anisotropy. Algorithm 3 outlines the algorithm for smooth anisotropic mean curvature flow using the inverse scale space flow (5.2).

REMARK 5.2. The trivial modification to the inverse scale space method for the evolution PDE (1.6) with the mobility function $\beta=\gamma$ is to replace the first differential equation in (5.2) by

$$
\frac{\partial \theta}{\partial s}=\frac{p^{\perp} \cdot \nabla u}{\gamma(\theta)\left[\gamma(\theta)+\gamma^{\prime \prime}(\theta)\right]}
$$

for which the preceding results hold true. 
Input: $u_{0}=\operatorname{dist}\left(S^{0}\right)$, final time $T>0$, time step $h>0$.

Output: $u_{[T / h]}=\operatorname{dist}\left(S_{h}(T)\right)$.

foreach $j=0,1, \ldots,[T / h]-1$ do

Set $f:=u_{j}$;

Initialize $\theta(0):=\tan ^{-1}\left(-f_{x} / f_{y}\right), u(0):=f$;

Solve (5.2) until steady state: $u^{*}:=\lim _{s \rightarrow \infty} u(s)$;

Set $u_{j+1}:=\operatorname{dist}\left(\left\{u^{*} \leq 0\right\}\right)$;

end

Algorithm 3: Anisotropic mean curvature flow using the inverse scale space flow.

\section{Numerical results}

6.1. Crystalline mean curvature flow. We numerically solved the anisotropic mean curvature flow using the polyhedral shrinkage algorithm (Algorithm 2) within Algorithm 1. The boundary value problem (3.10), (3.11) for $u^{k+1}$ was solved using a standard finite difference discretization on a uniform grid. The distance functions were computed using the fast sweeping method [49]; more accurate computations of the signed distance function along the zero level set can be computed using numerical PDE techniques, c.f. [14].
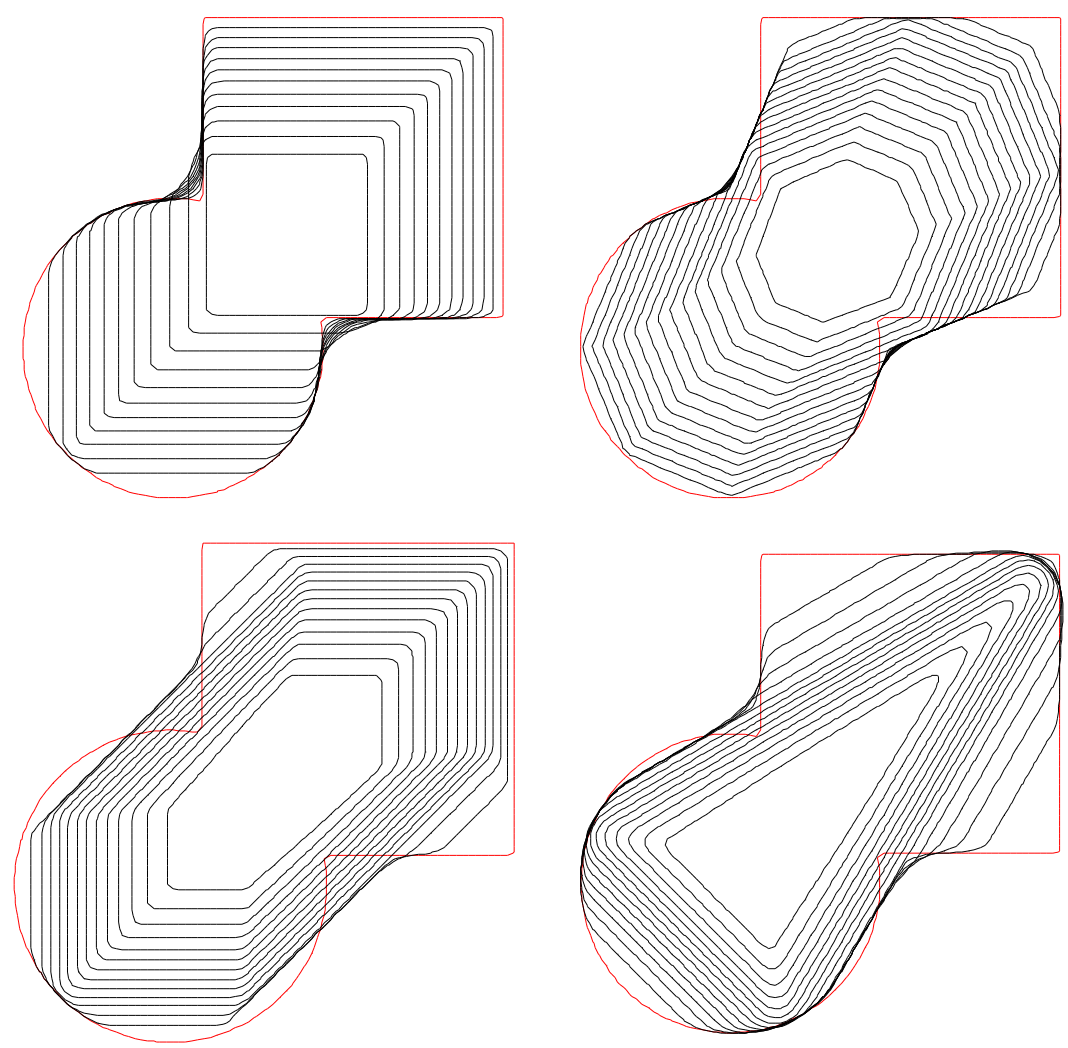

Fig. 6.1: Crystalline mean curvature motion for various anisotropies. Clockwise from top left: $\mathcal{N}_{1}, \mathcal{N}_{8}, \mathcal{N}_{\text {tri }}$, and $\mathcal{N}_{\text {skew }}$. 

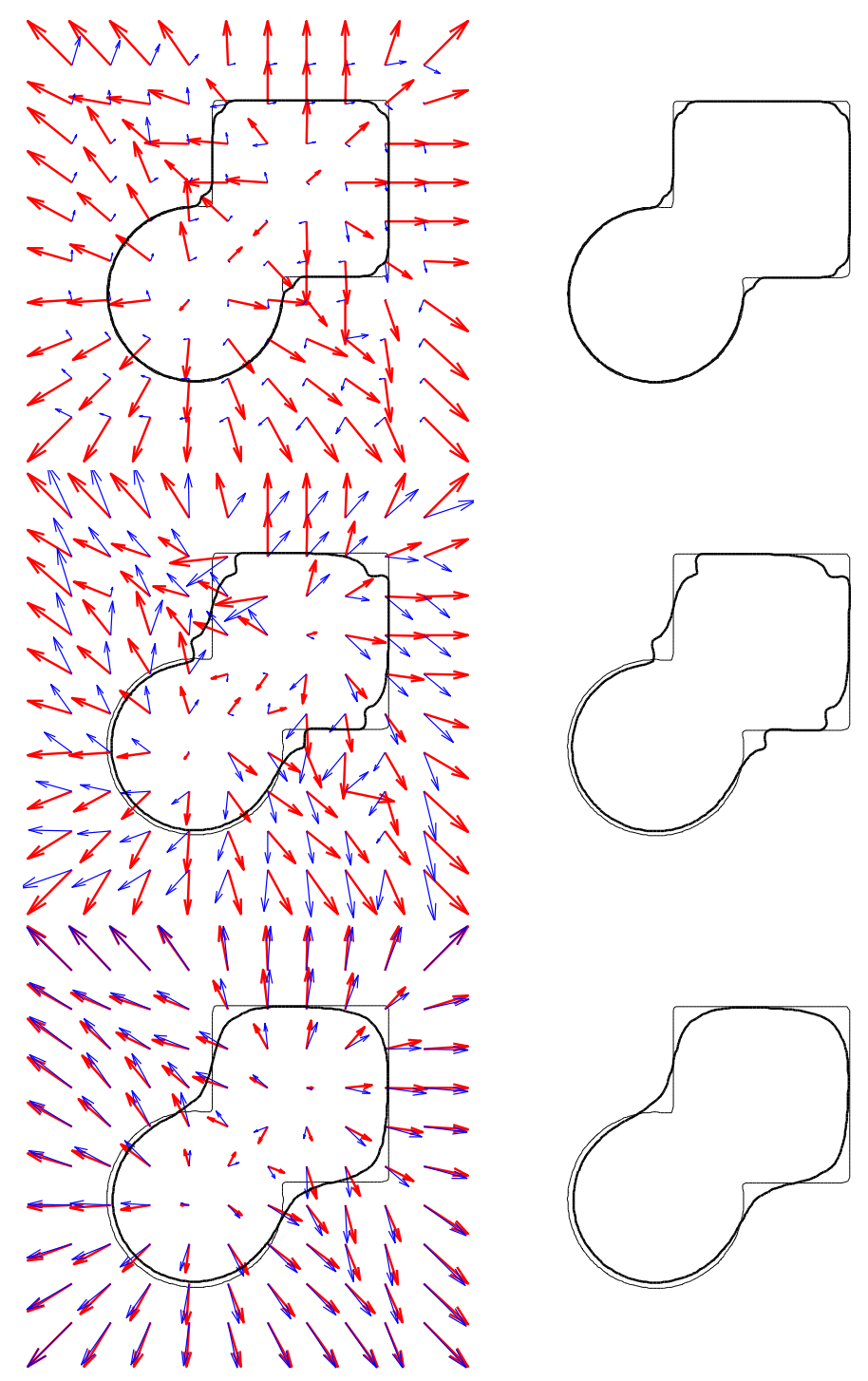

Fig. 6.2: Visualization of $\nabla u$ and $d$ in the inverse scale space flow (5.2), with $\gamma_{0}=1$ (the isotropic case). Thick arrows are $\nabla u$ and the thin arrows are $d$. Top to bottom: 200, 1000, 3000 iterations.

The norms (4.5) considered were:

- 1-norm: $\mathcal{N}_{1}=\{ \pm(1,1), \pm(1,-1)\}$.

- Octagon norm: $\mathcal{N}_{8}=\{( \pm 1,0),(0, \pm 1),( \pm 1, \pm 1) / \sqrt{2},( \pm 1, \mp 1) / \sqrt{2}\}$.

- A skewed hexagon norm: $\left.\mathcal{N}_{\text {skew }}=\{( \pm 1,0),(0, \pm 1), \pm(1,1))\right\}$.

- A triangle 'norm': $\mathcal{N}_{t r i}=\{(\sqrt{2}, \sqrt{2}),(-1,0),(0,-1)\}$.

Figure 6.1 shows the results for these anisotropies on a $100 \times 100$ grid with $h=$ 0.015 . The outer and inner iterations were computed until consecutive approximations were bounded by $2 \mathrm{e}-5$. Typically, it took less than 20 outer iterations, while the inner iterations ranged from 2 to 500. Notice the emergence of the Wulff shapes as the 


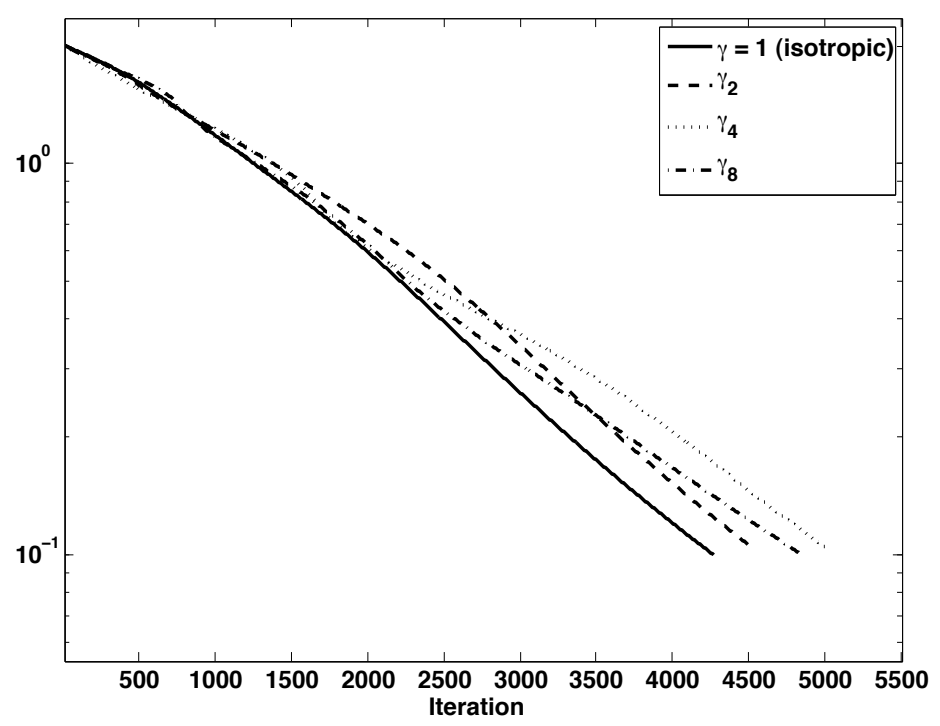

Fig. 6.3: Plot of $\|\nabla u-d\|$ in a log-scale over iterations in the inverse scale space variable $s$.

curves near extinction; see Appendix A for Wulff shapes of polyhedral norms. Note that $\mathcal{N}_{\text {tri }}$ does not induce a norm due to its asymmetry, but the algorithm computes the flow nonetheless.

6.2. Inverse scale space algorithm. We first present a sequence of plots showing how the inverse scale space flow (5.2) behaves. For the isotropic case $(\gamma=1)$ with time step $h=0.015$, Figure 6.2 shows $\nabla u(s), d(s)$, and the zero level set of $u(s)$ at various stages in the iteration over $s$, where $\Delta s=0.001$. Note how $\theta(s)$ (the direction of the vector $d(s))$ moves clockwise as per result 4, Lemma 5.3. The stopping condition was $\left\|u^{n+1}-u^{n}\right\|_{\infty} \leq 1 \mathrm{e}-5$, where $u^{n}$ is the numerical approximation of $u(n \Delta s)$, for which the iteration halted after 3770 iterations. We have observed that the number of iterations necessary for convergence (for the same stopping condition and time step) was roughly between 3000 to 4000 for grid sizes $25^{2}, 50^{2}, 100^{2}$, and $150^{2}$ on the square $\Omega=[-1,1]^{2}$. It is reasonable to believe that the number of iterations required for convergence should be independent of the grid size, since convergence is attained for a large enough $s$, i.e. solely dependent on the underlying equation (i.e. choice of $\gamma$, $f)$.

Figure 6.3 shows a semi-logarithmic plot of $\|\nabla u(s)-d(s)\|_{L^{2}(\Omega)}$ over iterations in $s$ of $(5.2)$, for various anisotropies. The anisotropies tested were

$$
\gamma(\omega)=\gamma_{n}(\omega):=\frac{1}{n^{2}+1}\left(n^{2}+1-\sin (n \omega)\right), \quad \text { for } n=0,2,4,8
$$

where $\gamma(\omega)$ is defined in (5.1). Note that $n=0$ is the isotropic case; for $n=2,4,8$, the anisotropy has a $n$-fold rotational symmetry. We have found that, in general, the isotropic case converges faster to steady state than the anisotropic cases; also, for all cases tested, $\|\nabla u-d\|_{L^{2}(\Omega)}$ eventually converges exponentially, corroborating with the results of Proposition 5.1.

Results for Algorithm 3 are shown in Figure 6.4 for the anisotropies (6.1). 

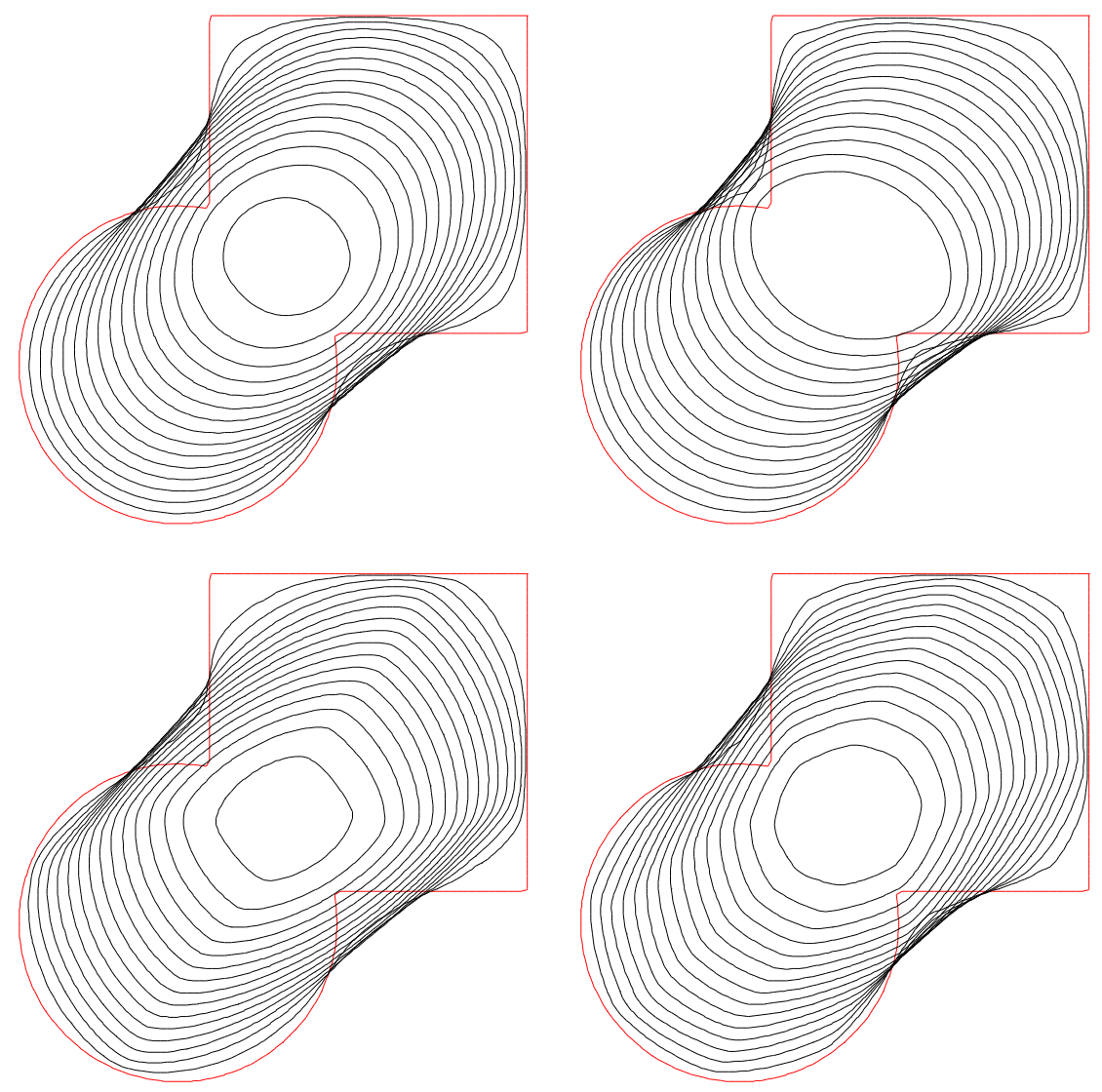

Fig. 6.4: Mean curvature motion for various smooth anisotropies. Clockwise from top left: $\gamma_{0}$ (isotropic), $\gamma_{2}, \gamma_{8}$, and $\gamma_{4}$.

\section{Conclusions}

We have presented new methods of computing crystalline and smooth anisotropic mean curvature flows. In the crystalline case, we proved a formula for the anisotropic shrinkage problem, which was embedded in the split Bregman framework for mean curvature flow. In the smooth anisotropy case, we formally derived an inverse scale space flow, which, in the steady state limit, solves the semi-implicit Euler scheme of the mean curvature PDE. Numerical results for both methods were presented.

Acknowledgement. S. Osher and R. Takei were partially supported by ONR grants N00014-03-1-007 and N00014-07-0810. R. Tsai was partially supported by NSF grant DMS-0714612. The authors thank M. Moeller for proofreading parts of an earlier draft and the anonymous reviewers for their constructive suggestions.

Appendix A. Formulae for Wulff shapes. Assume that $\phi: \mathbb{R}^{2} \rightarrow \mathbb{R}$ is a convex, positively 1-homogenous function.

The formulas for the Wulff shapes are defined implicitly through the dual norms. However, when we have explicit polyhedral formulas for the norms, we can also obtain explicit formulas for the Wulff shapes. 
Proposition A.1. Let $\phi$ be a polyhedral norm, written explicitly as

$$
\phi(x)=\max _{n_{i} \in \mathcal{N}} n_{i} \cdot x,
$$

$\left(\mathcal{N}\right.$ is the set of normals, as in (4.5)) and let $\phi^{\circ}$ be its dual norm. The Wulff shape is the polygon with vertices $\mathcal{N}$ :

$$
\mathcal{W}_{\phi}=\operatorname{conv}(\mathcal{N})
$$

Proof. We show that $\phi^{\circ}(x)=1$ on the boundary of the polygon $\operatorname{conv}(\mathcal{N})$. The result follows since $\phi^{\circ}$ is positively 1-homogeneous. From the definition, for an arbitrary $n_{j} \in \mathcal{N}$,

$$
\phi^{\circ}\left(n_{j}\right)=\max \left\{n_{j} \cdot y \mid \max _{n_{i} \in \mathcal{N}}\left\{n_{i} \cdot y\right\}=1\right\} \leq 1 .
$$

By choosing $y=n_{j} /\left|n_{j}\right|$, we see that $\phi^{\circ}\left(n_{j}\right)=1$. Next, for $\lambda \in(0,1)$ and two adjacent normals $n_{j}, n_{j+1} \in \mathcal{N}$,

$$
\phi^{\circ}\left(n_{j} \lambda+n_{j+1}(1-\lambda)\right)=\max \left\{n_{j} \cdot y \lambda+n_{j+1} \cdot y(1-\lambda) \mid \max _{n_{i} \in \mathcal{N}}\left\{n_{i} \cdot y\right\}=1\right\} .
$$

The maximizing $y$ satisfies $n_{j} \cdot y=n_{j+1} \cdot y=1$. Therefore, $\phi^{\circ}\left(n_{j} \lambda+n_{j+1}(1-\lambda)\right)=\lambda+$ $(1-\lambda)=1$.

\section{Appendix B. Boundary conditions for the Euler-Lagrange Equation} (3.10).

We derive the boundary conditions (3.11), for the minimization

$$
\min _{u} I(u):=\frac{\lambda}{2} \int_{\Omega}(u-f)^{2}+\frac{\mu}{2} \int_{\Omega}\left|d^{k}-\nabla u-b^{k}\right|^{2},
$$

for fixed functions $d^{k}, b^{k}, f$ and constants $\lambda, \mu>0$. To this end, we follow the standard arguments for deriving the first variation; c.f. [24, Chapter 8]: assume $u^{k+1}$ is the minimizer of $I(u)$ and choose any smooth function $v \in C^{\infty}(\Omega)$. Set

$$
i(\tau):=I\left(u^{k+1}+\tau v\right), \quad \tau \in \mathbb{R} .
$$

By the necessary condition of the minimizer,

$$
0=i^{\prime}(0)=\lambda \int_{\Omega}\left(u^{k+1}-f\right) v+\mu \int_{\Omega}\left(d^{k}-\nabla u^{k+1}-b^{k}\right)(-\nabla v) .
$$

Furthermore, integrating the second term by parts,

$$
0=\lambda \int_{\Omega}\left(u^{k+1}-f\right) v+\mu \int_{\Omega} \operatorname{div}\left(d^{k}-\nabla u^{k+1}-b^{k}\right) v-\mu \int_{\partial \Omega}\left(d^{k}-\nabla u^{k+1}-b^{k}\right) \cdot n v
$$

where $n$ is the unit outer normal of $\partial \Omega$. The first two terms in (B.1) yields the Euler-Lagrange equation (3.10), while setting the last term to zero gives the desired boundary condition (3.11). 


\section{REFERENCES}

[1] F. Almgren, J.E. Taylor, and L. Wang, Curvature-driven flows: A variational approach, SIAM J. Control Optim., 31(2), 387-438, 1993.

[2] G. Bellettini, A sampler of Riemann-Finsler geometry, MSRI Publications, Camgridge University Press, 50, 49-82, 2004.

[3] M. Burger, G. Gilboa, S. Osher, and J. Xu, Nonlinear inverse scale space methods, Commun. Math. Sci., 4(1), 179-212, 2006.

[4] L.M. Brègman, A relaxation method of finding a common point of convex sets and its application to the solution of problems in convex programming, Z̆. Vyčisl. Mat. i Mat. Fiz., 7, 620-631, 1967.

[5] M. Burger, C. Stöcker, and A. Voigt, Finite element-based level set methods for higher order flows, J. Sci. Comput., 35(2-3), 77-98, 2008.

[6] A. Chambolle and J. Darbon, On total variation minimization and surface evolution using parametric maximum flows, Int. J. Comput. Vision, 84(3), 288-307, 2009.

[7] F. Catté, F. Dibos, and G. Koepfler, A morphological scheme for mean curvature motion and applications to anisotropic diffusion and motion of level sets, SIAM J. Numer. Anal., 32(6), 1895-1909, 1995.

[8] Y.G. Chen, Y. Giga, and S. Goto, Uniqueness and existence of viscosity solutions of generalized mean curvature flow equations, J. Differ. Geom., 33(3), 749-786, 1991.

[9] A. Chambolle, An algorithm for mean curvature motion, Interfaces Free Bound., 6(2), 195-218, 2004.

[10] A. Chambolle, An algorithm for total variation minimization and applications, J. Math. Imaging Vision, 20(1-2), 89-97, 2004. Special Issue on Mathematics and Image Analysis.

[11] U. Clarenz, F. Haußer, M. Rumpf, A. Voigt, and U. Weikard, On level set formulations for anisotropic mean curvature flow and surface diffusion, in Multiscale Modeling in Epitaxial Growth, Internat. Ser. Numer. Math., Birkhäuser, Basel, 149, 227-237, 2005.

[12] A. Chambolle and M. Novaga, Convergence of an algorithm for the anisotropic and crystalline mean curvature flow, SIAM J. Math. Anal., 37(6), 1978-1987, 2006.

[13] A. Chambolle and M. Novaga, Approximation of the anisotropic mean curvature flow, Math. Mode. Meth. Appl. Sci., 17(6), 833-844, 2007.

[14] L.-T. Cheng and Y.-H. Tsai, Redistancing by flow of time dependent eikonal equation, J. Comput. Phys., 227(8), 4002-4017, 2008.

[15] K. Deckelnick, G. Dziuk, and C.M. Elliott, Computation of geometric partial differential equations and mean curvature flow, Acta Numer., 14, 139-232, 2005.

[16] C. Dohmen, Y. Giga, and N. Mizoguchi, Existence of selfsimilar shrinking curves for anisotropic curvature flow equations, Calc. Var. Part. Differ. Eqs., 4(2), 103-119, 1996.

[17] J. Eckstein and D.P. Bertsekas, On the Douglas-Rachford splitting method and the proximal point algorithm for maximal monotone operators, Math. Programming, 55(3), 293-318, 1992.

[18] S. Esedoḡlu and S.J. Osher, Decomposition of images by the anisotropic Rudin-Osher-Fatemi model, Commun. Pure Appl. Math., 57(12), 1609-1626, 2004.

[19] S. Esedoḡlu, S. Ruuth, and R. Tsai, Threshold dynamics for shape reconstruction and disocclusion, Proc. Int. Conf. Image Processing, 502-505, 2005.

[20] S. Esedoğlu, S. Ruuth, and R. Tsai, Threshold dynamics for high order geometric motions, Interfaces and Free Boundaries, 10(3), 2008.

[21] S. Esedoḡlu, S. Ruuth, and R. Tsai, Diffusion generated motion using signed distance functions, J. Comput. Phys., 229(4), 1017-1042, 2010.

[22] L.C. Evans and J. Spruck, Motion of level sets by mean curvature. I, J. Diff. Geom., 33(3), 635-681, 1991.

[23] S. Esedoglu and Y.-H.R. Tsai. Threshold dynamics for the piecewise constant Mumford-Shah functional, J. Comput. Phys., 211(1), 367-384, 2006.

[24] L.C. Evans, Partial Differential Equations, Graduate Studies in Mathematics, 19, American Mathematical Society, Providence, RI, second edition, 2010.

[25] T. Goldstein, X. Bresson, and S. Osher, Geometric applications of the split Bregman method: Segmentation and surface reconstruction, Technical report, UCLA, ftp://ftp.math.ucla.edu/pub/camreport/cam09-06.pdf, July, 2009.

[26] R. Grzhibovskis and A. Heintz, A convolution thresholding scheme for the willmore flow, Interfaces and Free Boundaries, 10(2), 139-153, 2008.

[27] Y. Giga, Surface evolution equations, a level set approach, Monographs in Mathematics, Birkhäuser Verlag, Basel, 99, 2006.

[28] M.E. Gage and Y. Li, Evolving plane curves by curvature in relative geometries. II, Duke Math. 
J., 75(1),79-98, 1994.

[29] R. Glowinski and A. Marrocco, Sur l'approximation, par éléments finis d'ordre un, et la résolution, par pénalisation-dualité, d'une classe de problèmes de Dirichlet non linéaires, Rev. Française Automat. Informat. Recherche Opérationnelle RAIRO Analyse Numérique, 9(R-2), 41-76, 1975.

[30] D. Gabay and B. Mercier, A dual algorithm for the solution of nonlinear variational problems via finite element approximation, Comput. Math. Appl., 2, 17-40, 1976.

[31] T. Goldstein and S. Osher, The split Bregman method for L1-regularized problems, SIAM J. Imaging Sci., 2(2), 323-343, 2009.

[32] M.E. Gurtin, Thermomechanics of Evolving Phase Boundaries in The Plane, Oxford Mathematical Monographs, The Clarendon Press Oxford University Press, New York, 1993.

[33] F. Haußer and A. Voigt, A numerical scheme for regularized anisotropic curve shortening flow, Appl. Math. Lett., 19(8), 691-698, 2006.

[34] H. Ishii, G.E. Pires, and P.E. Souganidis, Threshold dynamics type approximation schemes for propagating fronts, J. Math. Soc. Japan, 51(2), 267-308, 1999.

[35] S. Luckhaus and T. Sturzenhecker, Implicit time discretization for the mean curvature flow equation, Calc. Var. Part. Differ. Eqs., 3(2), 253-271, 1995.

[36] Y. Lucet, Faster than the fast Legendre transform, the linear-time Legendre transform, Numer. Algorithms, 16(2), 171-185, 1998.

[37] B. Merriman, J.K. Bence, and S.J. Osher, Motion of multiple functions: A level set approach, J. Comput. Phys., 112(2), 334-363, 1994.

[38] M. Novaga and M. Paolini, Nonuniqueness for crystalline curvature flow, Math. Mode. Meth. Appl. Sci., 17(8), 1307-1315, 2007.

[39] J. Nocedal and S.J. Wright, Numerical Optimization, in Springer Series in Operations Research and Financial Engineering, Springer, New York, second edition, 2006.

[40] A.M. Oberman, A convergent monotone difference scheme for motion of level sets by mean curvature, Numer. Math., 99(2), 365-379, 2004.

[41] S. Osher, M. Burger, D. Goldfarb, J. Xu, and W. Yin, An iterative regularization method for total variation-based image restoration, Multiscale Model. Simul., 4(2), 460-489 (electronic), 2005.

[42] S. Osher and J.A. Sethian, Fronts propagating with curvature-dependent speed: algorithms based on Hamilton-Jacobi formulations, J. Comput. Phys., 79(1), 12-49, 1988.

[43] S.J. Ruuth and B. Merriman, Convolution-generated motion and generalized Huygens' principles for interface motion, SIAM J. Appl. Math., 60(3), 868-890, 2000.

[44] L. Rudin, S. Osher, and E. Fatemi, Reconstruction and enhancement of signals using nonlinear non-oscillatory variational methods, Phys. D, 60(1-4), 259-268, 1992.

[45] S.J. Ruuth, A diffusion-generated approach to multiphase motion, J. Comput. Phys., 145(1), 166-192, 1998

[46] J.A. Sethian, Level Set Methods and Fast Marching Methods, in Cambridge Monographs on Applied and Computational Mathematics, Cambridge University Press, Cambridge, second edition, 3, 1999. Evolving interfaces in computational geometry, fluid mechanics, computer vision, and materials science.

[47] S. Setzer, Split Bregman algorithm, Douglas-Rachford splitting and frame shrinkage, X.C. Tai, K. Morken, M. Lysaker, and K.A. Lie, editors, in Scale Space and Variational Methods in Computer Vision, SSVM, Springer Berlin / Heidelberg, July, 464-476, 2009.

[48] J.E. Taylor, Anisotropic interface motion, L.-Q. Chen, B. Fultz, J.W. Cahn, J.R. Manning, J.E. Morral, and J. Simmons, editors, in Mathematics of Microstructure Evolution, SIAM, 4, 135-148, 1996.

[49] Y.-H.R. Tsai, L.-T. Cheng, S. Osher, and H.-K. Zhao, Fast sweeping algorithms for a class of Hamilton-Jacobi equations, SIAM J. Numer. Anal., 41(2), 673-694 (electronic), 2003.

[50] Y.-H.R. Tsai, Rapid and accurate computation of the distance function using grids, J. Comput. Phys., 178, 175-195, 2002. 